\title{
Stratifications and Mackey Functors I: Functors for a Single Group
}

\author{
Peter Webb* \\ School of Mathematics, University of Minnesota, Minneapolis MN 55455, USA \\ email: webb@math.umn.edu
}

\begin{abstract}
In the context of Mackey functors we introduce a category which is analogous to the category of modules for a quasi-hereditary algebra which have a filtration by standard objects. Many of the constructions which work for quasi-hereditary algebras can be done in this new context. In particular we construct an analogue of the 'Ringel dual', which turns out here to be a standardly stratified algebra. The Mackey functors which play the role of the standard objects are constructed in the same way as functors which have been previously used in parametrizing the simple Mackey functors, but instead of using simple modules in their construction (as was done before) we use $p$-permutation modules. These Mackey functors are obtained as adjoints of the operations of forming the Brauer quotient and its dual. The filtrations which have these Mackey functors as their factors are closely related to the filtrations whose terms are the sum of induction maps from specified subgroups, or are the common kernel of restriction maps to these subgroups. These latter filtrations appear in Conlon's decomposition theorems for the Green ring, as well as in other places, where they arise quite naturally.
\end{abstract}

* Partially supported by the NSF. 


\section{Introduction}

This work is a study of several fundamental constructions in representation theory and their interactions in the context of Mackey functors. There are really four main ingredients and we will now describe them.

The first ingredient is a pair of filtrations of each Mackey functor $M$ for a finite group $G$. These are constructed by considering for each family of subgroups $\mathcal{X}$, closed under conjugation and taking subgroups, at each subgroup $H$ the sum of the images in $M(H)$ of the inductions from subgroups in $\mathcal{X}$, and the intersection of the kernels of the restrictions to subgroups in $\mathcal{X}$. The filtrations are constructed by enlarging $\mathcal{X}$ by adjoining one conjugacy class of subgroups at a time. We call the two filtrations the ascending and descending filtrations of $M$ associated to the order in which the conjugacy classes of subgroups of $G$ are taken. Such constructions have appeared widely before; for example, they underlie Conlon's theorems on the Green ring [5, Section 81], and also one of the proofs of semisimplicity of Mackey functors when $|G|$ is invertible [13]. This in turn makes use of a theorem of Dress [6, Theorems 2 and 4] which really has to do with these filtrations.

The second ingredient in our work is the notion of the Brauer quotient, and its dual, the restriction kernel. Brauer quotients have played a fundamental role in recent representation theory, as described in [12]. The restriction kernel has appeared less overtly, in spite of the fact that over a field it is the dual of the Brauer quotient applied to the dual Mackey functor. It appears explicitly in a conjecture of Feshbach (see [1]) where it is called the essential elements. The Brauer quotient and restriction kernel turn out to be certain values of the factors in ascending and descending filtrations of the Mackey functor.

The next ingredient is a pair of Mackey functors $\Delta_{H, U}$ and $\nabla_{H, U}$ associated to each subgroup $H$ and $N_{G}(H) / H$-representation $U$. The Mackey functors $\nabla_{H, U}$ were first defined by Lewis [8], and they also appeared without special terminology in [13] where it was shown that when $U$ is a simple module, $\nabla_{H, U}$ has a unique simple subfunctor. Arising from this a complete parametrization of the simple Mackey functors was obtained. It is because of this property that we use the $\nabla$ notation here. Similarly, when $U$ is simple, $\Delta_{H, U}$ has a unique simple quotient Mackey functor. However, we should point out that the notation is flawed in that not all of our functors $\nabla_{H, U}$ have a simple socle, and neither do all of the functors $\Delta_{H, U}$ have a simple top. An indication of the fundamental nature of these Mackey functors is given by the fact that the functor $U \mapsto \nabla_{H, U}$ is the right adjoint of the functor which assigns to a Mackey functor $M$ its Brauer quotient at $H$; and the functor $U \mapsto \Delta_{H, U}$ is the left adjoint of the functor which assigns to $M$ its restriction kernel at $H$. Indeed, these properties may be used to define these Mackey functors.

The fourth main ingredient in our work is the class of $p$-permutation modules, by which we mean modules which are direct summands of permutation modules. Their appearance here is not accidental, and really derives from the fact that if $M$ is a projective Mackey functor, then all of its Brauer quotients are $p$-permutation modules (or zero). We will deal

with the functors $\Delta_{H, U}$ and $\nabla_{H, U}$ where $U$ is a $p$-permutation module for $N_{G}(H) / H$. 
One of the main objects of our study will be the full subcategory $\mathcal{D}$ of the category of all Mackey functors for $G$ whose objects are the Mackey functors which have a filtration whose factors are of the form $\Delta_{H, U}$ with $U$ a $p$-permutation module. Such a filtration we will call a $\Delta$-filtration. There is also an analogous category whose objects have a $\nabla$-filtration, and the results we prove for $\mathcal{D}$ have dual statements for this other category. We will show that the projective Mackey functors are objects in $\mathcal{D}$ (Theorem 7.1). The ascending filtrations have an importance for the objects of $\mathcal{D}$ in that any ascending filtration of an object in $\mathcal{D}$ can be refined to a $\Delta$-filtration (Proposition 6.1). We follow the direction of the work of Ringel [10] and we prove a theorem on the vanishing of Ext groups between the $\Delta_{H, U}$ (Theorem 5.1). This allows us to invoke Ringel's work and deduce that $\mathcal{D}$ is functorially finite in the full category of Mackey functors and has relative almost split sequences. We go on to investigate the relative Auslander-Reiten quiver for $\mathcal{D}$, and make several characterizations of particular Mackey functors in $\mathcal{D}$ (Section 11); but most importantly we characterize the Ext-projective and Ext-injective objects in $\mathcal{D}$. This is done through an axiomatic notion of Ext-projective covers and Ext-injective hulls developed in Section 8. The work in Section 8 applies in greater generality than the context of Mackey functors, and may be of separate interest. The indecomposable Ext-projective objects and Ext-injective objects in $\mathcal{D}$ are in fact precisely the Ext-projective covers $\Pi_{H, U}$ and the Ext-injective hulls $T_{H, U}$ of the $\Delta_{H, U}$. We now do what Ringel did and form the algebra $E=\operatorname{End}\left(\bigoplus_{H, U} T_{H, U}\right)$. Since we are not working with a quasi hereditary algebra here (in contrast to Ringel's situation) we do not expect $E$ to be quasi-hereditary. It is, however, standardly stratified in the sense of [4] with respect to an obvious pre-order on the set of pairs $(H, U)$ which parametrize the simple $E$-modules. The algebra $E$ stands with respect to the group algebra of $G$ in the same way as a Schur algebra stands with respect to the group algebra of a symmetric group, in that the group algebra is Morita equivalent to $e E e$ for a certain idempotent $e$.

We will use the notation and basic constructions which appear in [14]. Indeed, many of the main ideas of [14] are used throughout this work and a good acqaintance with these ideas is really a prerequisite to understanding some of our arguments. Let us summarize the most basic notation. Unless otherwise stated we will be working with Mackey functors defined on a finite group $G$ and its subgroups. Thus when $H$ is a subgroup of $G$ and $M$ is a Mackey functor we have operations $I_{H}^{G}: M(H) \rightarrow M(G)$ and $R_{H}^{G}: M(G) \rightarrow M(H)$ which behave like induction and restriction in representation theory, and for each $g \in G$ we have a conjugation morphism $c_{g}: M(H) \rightarrow M\left({ }^{g} H\right)$. We will always work with Mackey functors over a commutative ring $R$ with a 1 , and in the later parts of this paper we will require that $R$ be a complete p-local ring, by which we mean that $R$ is either a field of characteristic $p$ or a complete discrete valuation ring with residue field of characteristic $p$.

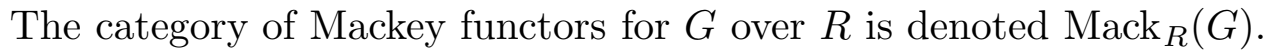

I wish to thank the referee for his or her careful reading of the manuscript and helpful comments. 


\section{Filtrations, Brauer quotients and restriction kernels}

We start by describing the subfunctors of an arbitrary Mackey functor $M$ which will form the terms of its ascending and descending filtrations. For this we will need the notion of the subfunctor of $M$ generated by a collection of subsets $A_{H} \subseteq M(H)$, where $H$ ranges over (not necessarily all) subgroups of $G$. By this we mean the intersection of all subfunctors $N$ of $M$ for which $N(H) \supseteq A_{H}$ for all $H$, and it is the unique minimal subfunctor of $M$ containing all these subsets. A useful description of this subfunctor is stated in $[14,2.4]$.

Suppose that $\mathcal{X}$ is a set of subgroups of $G$. We define

$$
I_{\mathcal{X}} M=\langle M(J) \mid J \in \mathcal{X}\rangle
$$

to be the subfunctor of $M$ generated by its values on groups in $\mathcal{X}$. We also define $R_{\mathcal{X}} M$ to be the largest subfunctor of $M$ satisfying the condition

$$
R_{J}^{K}: R_{\mathcal{X}} M(K) \rightarrow M(J) \text { is the zero map, for every group } J \in \mathcal{X} \text { and } K \supseteq J \text {. }
$$

Most of the time we will assume that $\mathcal{X}$ is closed under taking subgroups and conjugation, and in this context these functors were considered in [13, Sect. 3] where they were denoted $\operatorname{Im} t_{\mathcal{X}}$ and $\operatorname{Ker} r_{\mathcal{X}}$

We list some of the properties of these Mackey functors. In part (e) of our first result we use the term 'cogenerated' in a sense dual to that of the word 'generated'. We will say that a Mackey functor $M$ is cogenerated by subsets $A_{H} \subseteq M(H)$ if every morphism of Mackey functors $M \rightarrow M^{\prime}$ which is injective on all the subsets $A_{H}$ is in fact a monomorphism. In part (g) we refer to a construction introduced by Dress [6] in the study of relative projectivity, and at this point we use an alternative notation for Mackey functors in which they are defined on $G$-sets, rather than on subgroups of $G$ (see [14]). Whenever $X$ is a $G$-set and $M$ a Mackey functor we may define a Mackey functor $M_{X}$ whose value at a $G$-set $Y$ is $M(Y \times X)$. There is a natural transformation $\theta_{X}: M_{X} \rightarrow M$ which is determined at the $G$-set $Y$ by applying the covariant part of $M$ to projection onto the first coordinate, giving a homomorphism $M(Y \times X) \rightarrow M(Y)$. Similarly there is a natural transformation $\theta^{X}: M \rightarrow M_{X}$ determined by applying the contravariant part of $M$ to projection onto the first coordinate. Part ( $\mathrm{j}$ ) of the result is a special case of the induction theorem of Dress [6]. We do not give the complete formulation so as to save space, and we will not use it, but merely take the opportunity to point out this important result. 
(2.1) PROPOSITION. Let $\mathcal{X}, \mathcal{X}_{1}$ and $\mathcal{X}_{2}$ be sets of subgroups of $G$.

(a) $I_{\emptyset} M=R_{\text {all subgroups }} M=0$ and $R_{\emptyset} M=I_{\text {all subgroups }} M=M$.

(b) If $\mathcal{X}_{1} \subseteq \mathcal{X}_{2}$ then $I_{\mathcal{X}_{1}} M \subseteq I_{\mathcal{X}_{2}} M$ and $R_{\mathcal{X}_{1}} M \supseteq R_{\mathcal{X}_{2}} M$.

(c) Let $\mathcal{X}^{+}$be the closure of $\mathcal{X}$ under adjoining conjugates. Then $I_{\mathcal{X}} M=I_{\mathcal{X}} M$ and $R_{\mathcal{X}} M=R_{\mathcal{X}+} M$.

(d) If $\mathcal{X}$ is closed under taking subgroups and conjugation then

$$
I_{\mathcal{X}} M(K)=\sum_{K \supseteq L \in \mathcal{X}} I_{L}^{K}(M(L)) \quad \text { and } \quad R_{\mathcal{X}} M(K)=\bigcap_{K \supseteq L \in \mathcal{X}} \operatorname{Ker} R_{L}^{K}
$$

(e) If $\mathcal{X}_{1} \subseteq \mathcal{X}_{2}$ then $I_{\mathcal{X}_{2}} M / I_{\mathcal{X}_{1}} M$ is generated by its values on the subgroups in $\mathcal{X}_{2}-\mathcal{X}_{1}$ and $R_{\mathcal{X}_{1}} M / R_{\mathcal{X}_{2}} M$ is cogenerated by its values on the subgroups in $\mathcal{X}_{2}-\mathcal{X}_{1}$. If additionally $\mathcal{X}_{1}$ and $\mathcal{X}_{2}$ are closed under taking subgroups then $I_{\mathcal{X}_{2}} M / I_{\mathcal{X}_{1}} M$ and $R_{\mathcal{X}_{1}} M / R_{\mathcal{X}_{2}} M$ are zero on subgroups which do not contain a conjugate of a subgroup in $\mathcal{X}_{2}-\mathcal{X}_{1}$.

(f) $I_{\mathcal{X}}\left(M_{1} \oplus M_{2}\right)=I_{\mathcal{X}} M_{1} \oplus I_{\mathcal{X}} M_{2}$ and $R_{\mathcal{X}}\left(M_{1} \oplus M_{2}\right)=R_{\mathcal{X}} M_{1} \oplus R_{\mathcal{X}} M_{2}$.

(g) Suppose $\mathcal{X}$ is closed under taking subgroups. Put $X=\bigsqcup_{K \in \mathcal{X}} G / K$. Then $I_{\mathcal{X}} M$ equals the image of $\theta_{X}: M_{X} \rightarrow M$ and $R_{\mathcal{X}} M$ equals the kernel of $\theta^{X}: M \rightarrow M_{X}$.

(h) Suppose that $\mathcal{X}$ is closed under taking subgroups, and if $H$ is a subgroup of $G$ put

$$
\mathcal{X}_{H}=\left\{H \cap{ }^{g} K \mid K \in \mathcal{X}, g \in G\right\}
$$

a set of subgroups of $H$ also closed under taking subgroups. Then when $M$ is a Mackey functor for $H$ we have

$$
\begin{aligned}
I_{\mathcal{X}}\left(M \uparrow_{H}^{G}\right) & =I_{\mathcal{X}_{H}}\left(M \uparrow_{H}^{G}\right)=\left(I_{\mathcal{X}_{H}} M\right) \uparrow_{H}^{G}, \\
R_{\mathcal{X}}\left(M \uparrow_{H}^{G}\right) & =R_{\mathcal{X}_{H}}\left(M \uparrow_{H}^{G}\right)=\left(R_{\mathcal{X}_{H}} M\right) \uparrow_{H}^{G},
\end{aligned}
$$

and when $M$ is a Mackey functor for $G$ we have

$$
\begin{gathered}
\left(I_{\mathcal{X}} M\right) \downarrow_{H}^{G}=\left(I_{\mathcal{X}_{H}} M\right) \downarrow_{H}^{G}=I_{\mathcal{X}_{H}}\left(M \downarrow_{H}^{G}\right), \\
\left(R_{\mathcal{X}} M\right) \downarrow_{H}^{G}=\left(R_{\mathcal{X}_{H}} M\right) \downarrow_{H}^{G}=R_{\mathcal{X}_{H}}\left(M \downarrow_{H}^{G}\right) .
\end{gathered}
$$

(i) $([15,3.4 .2])$ If $\mathcal{X}_{1} \subseteq \mathcal{X}_{2}$ and $M$ is projective relative to a set of subgroups $\mathcal{Y}$ then $I_{\mathcal{X}_{2}} M / I_{\mathcal{X}_{1}} M$ and $R_{\mathcal{X}_{1}} M / R_{\mathcal{X}_{2}} M$ are both projective relative to $\mathcal{Y}$.

(j) (Dress [6]) $|G| \cdot M \subseteq I_{\mathcal{X}} M+R_{\mathcal{X}} M$ and $|G| \cdot\left(I_{\mathcal{X}} M \cap R_{\mathcal{X}} M\right)=0$.

Proof. (a) and (b) are self-evident.

For (c) we have in one direction that $I_{\mathcal{X}} M \subseteq I_{\mathcal{X}^{+}} M$ and $R_{\mathcal{X}} M \supseteq R_{\mathcal{X}^{+}} M$ by (b). On the other hand if $L \in \mathcal{X}^{+}$then $L={ }^{g} L_{1}$ for some $L_{1} \in \mathcal{X}$ and now $M(L)=c_{g} M\left(L_{1}\right)$ is the image under conjugation by $g$. Thus $M(L) \subseteq I_{\mathcal{X}} M(L)$ and so $I_{\mathcal{X}} M \subseteq I_{\mathcal{X}} M$. A similar argument shows that $R_{\mathcal{X}} M \supseteq R_{\mathcal{X}} M$.

Assertion (d) follows from [13, 2.1] and the comment at the start of Section 3 in [13]. 
In (e), we have from the definitions that $I_{\mathcal{X}_{2}} M$ is generated by $I_{\mathcal{X}_{1}} M$ and the values of $M$ on the subgroups in $\mathcal{X}_{2}-\mathcal{X}_{1}$. Hence $I_{\mathcal{X}_{2}} M / I_{\mathcal{X}_{1}} M$ is simply generated by its values on the subgroups in $\mathcal{X}_{2}-\mathcal{X}_{1}$. We leave the statement about cogeneration as an exercise. If now $\mathcal{X}_{1}$ and $\mathcal{X}_{2}$ are closed under taking subgroups then by (c) it suffices to assume that $\mathcal{X}_{1}$ and $\mathcal{X}_{2}$ are closed under conjugacy, and by $(\mathrm{d})$ we see that $I_{\mathcal{X}_{2}} M$ is zero on subgroups which do not contain a conjugate of a subgroup in $\mathcal{X}_{2}$ and hence the same is true of $I_{\mathcal{X}_{2}} M / I_{\mathcal{X}_{1}} M$. If a subgroup contains a conjugate of a subgroup in $\mathcal{X}_{2}$, but not a conjugate of a subgroup in $\mathcal{X}_{2}-\mathcal{X}_{1}$, then $I_{\mathcal{X}_{2}} M$ and $I_{\mathcal{X}_{1}} M$ take the same values on this subgroup by (d), and so the quotient functor is zero there. Hence the quotient functor is only non-zero on subgroups which contain a conjugate of a subgroup in $\mathcal{X}_{2}-\mathcal{X}_{1}$. Again we leave the statement about $R_{\mathcal{X}_{1}} M / R_{\mathcal{X}_{2}} M$ as an exercise.

(f) is immediate.

(g) From the definitions of $\theta_{X}$ and $\theta^{X}$ we see that if $X^{\prime}$ is another $G$-set which has the same isomorphism types of $G=$ orbits as $X$, but with different multiplicities, then $\theta_{X}$ and $\theta_{X^{\prime}}$ have the same image, and $\theta^{X}$ and $\theta^{X^{\prime}}$ have the same kernel. From this we see that $\operatorname{Im} \theta_{X}=\operatorname{Im} \theta_{X^{+}}$and $\operatorname{Ker} \theta^{X}=\operatorname{Ker} \theta^{X^{+}}$where $X^{+}=\bigsqcup_{K \in \mathcal{X}^{+}} G / K$, since enlarging $\mathcal{X}$ to include conjugate subgroups does not change the isomorphism types of orbits in the $G$-sets.

We have already seen that $I_{\mathcal{X}} M=I_{\mathcal{X}+} M$ and $R_{\mathcal{X}} M=R_{\mathcal{X}+} M$, and so it suffices to prove the result when $\mathcal{X}$ is closed under taking subgroups and conjugation. With this hypothesis the result was proved in $[15,3.4 .3]$ for $I_{\mathcal{X}} M$. We leave the rest of the details for $R_{\mathcal{X}} M$ to the reader.

(h) Let us put $X=\bigsqcup_{K \in \mathcal{X}} G / K$ as a $G$-set, and $X_{H}=\bigsqcup_{K \in \mathcal{X}_{H}} H / K$ as an $H$-set. Then $X_{H} \uparrow_{H}^{G}$ may be identified as the $G$-set $\bigsqcup_{K \in \mathcal{X}_{H}} G / K$. Since

$$
G / K \downarrow_{H}^{G}=\bigsqcup_{g \in[H \backslash G / K]} H / H \cap{ }^{g} K
$$

and $H \cap{ }^{g} K \in \mathcal{X}_{H}$ when $K \in \mathcal{X}$ we see that $X \downarrow_{H}^{G}, X_{H} \uparrow_{H}^{G} \downarrow_{H}^{G}$ and $X_{H}$ all contain the same isomorphism types of $H$-orbits, but perhaps with different multiplicities. We have that $I_{\mathcal{X}}\left(M \uparrow_{H}^{G}\right)$ is the image of $\theta_{X}:\left(M \uparrow_{H}^{G}\right)_{X} \rightarrow M \uparrow_{H}^{G}$. At a $G$-set $G / L$ we have

$$
\begin{aligned}
& \left(M \uparrow_{H}^{G}\right)_{X}(G / L)=M \uparrow_{H}^{G}(G / L \times X) \\
& =M\left((G / L \times X) \downarrow_{H}^{G}\right) \\
& =M\left(G / L \downarrow_{H}^{G} \times X \downarrow_{H}^{G}\right) \\
& =M_{X \downarrow_{H}^{G}} \uparrow_{H}^{G}(G / L)
\end{aligned}
$$

so that $\left(M \uparrow_{H}^{G}\right)_{X} \cong M_{X \downarrow_{H}^{G}} \uparrow_{H}^{G}$, and with this identification $\theta_{X}$ becomes

$$
\left(\theta_{X \downarrow_{H}^{G}}\right) \uparrow_{H}^{G}: M_{X \downarrow_{H}^{G}} \uparrow_{H}^{G} \rightarrow M \uparrow_{H}^{G} .
$$


Since $\uparrow_{H}^{G}$ is an exact functor we see that $I_{\mathcal{X}}\left(M \uparrow_{H}^{G}\right)=\left(I_{\mathcal{X}_{H}} M\right) \uparrow_{H}^{G}$ since $X \downarrow_{H}^{G}$ has the same orbits as $X_{H}$. We may do the same calculation with $X_{H} \uparrow_{H}^{G}$ instead of $X$, to prove that $I_{\mathcal{X}_{H}}\left(M \uparrow_{H}^{G}\right)=\left(I_{\mathcal{X}_{H}} M\right) \uparrow_{H}^{G}$. This establishes the first line of equalities for $I_{\mathcal{X}}$, and the second line for $R_{\mathcal{X}}$ is proved similarly.

It is immediate from (d) that if $K \supseteq H$ then

$$
\left(I_{\mathcal{X}} M\right)(K)=\left(I_{\mathcal{X}_{H}} M\right)(K)=\left(I_{\mathcal{X}_{H}} M \downarrow_{H}^{G}\right)(K)
$$

which shows that

$$
\left(I_{\mathcal{X}} M\right) \downarrow_{H}^{G}=\left(I_{\mathcal{X}_{H}} M\right) \downarrow_{H}^{G}=I_{\mathcal{X}_{H}}\left(M \downarrow_{H}^{G}\right),
$$

and similarly with $R_{\mathcal{X}} M$.

For (i) we quote Proposition 3.4.2 from [15] (which uses different notation). A first application of that result shows that $I_{\mathcal{X}_{2}} M$ is projective relative to $\mathcal{Y}$. Then a second application shows that $I_{\mathcal{X}_{2}} M / I_{\mathcal{X}_{1}} M$ is projective relative to $\mathcal{Y}$. We may prove the result for $R_{\mathcal{X}_{1}} M / R_{\mathcal{X}_{2}} M$ by an analogous argument; or when $R$ is a field we may deduce it from what we have already done by duality using the result in the next proposition.

Over a field, the subfunctors $I_{\mathcal{X}} M$ and $R_{\mathcal{X}} M$ are in fact dual to each other in the sense that doing one of these to $M$ is equivalent to doing the other to the dual of $M$. When $R$ is a field, recall from [14, Sect. 4] that for each Mackey functor $M$ we define the dual Mackey functor $M^{*}$ by $M^{*}(K)=\operatorname{Hom}_{R}(M(K), R)$ with operations $R_{J}^{K}=\left(I_{J}^{K}\right)^{*}: M^{*}(K) \rightarrow$ $M^{*}(J), I_{J}^{K}=\left(R_{J}^{K}\right)^{*}: M^{*}(J) \rightarrow M^{*}(K)$ and conjugations $c_{g}=\left(c_{g^{-1}}\right)^{*}: M^{*}(J) \rightarrow$ $M^{*}\left({ }^{g} J\right)$. If $N \subseteq M$ is a subfunctor, let us put $N^{\perp}(K)=\left\{f \in M^{*}(K)|f|_{N(K)}=0\right\}$. Then by a standard piece of linear algebra if $N_{1} \subseteq N_{2} \subseteq M$ we have $\left(N_{2} / N_{1}\right)^{*} \cong N_{1}^{\perp} / N_{2}^{\perp}$.

(2.2) PROPOSITION. Let $M$ be a Mackey functor defined over a field and let $\mathcal{X}, \mathcal{X}_{1}$ and $\mathcal{X}_{2}$ be sets of subgroups of $G$ closed under conjugation and taking subgroups. Then $\left(I_{\mathcal{X}} M\right)^{\perp}=R_{\mathcal{X}}\left(M^{*}\right)$ and $\left(R_{\mathcal{X}} M\right)^{\perp}=I_{\mathcal{X}}\left(M^{*}\right)$. Thus if $\mathcal{X}_{1} \subseteq \mathcal{X}_{2}$ we have

$$
\left(I_{\mathcal{X}_{2}} M / I_{\mathcal{X}_{1}} M\right)^{*} \cong\left(I_{\mathcal{X}_{1}} M\right)^{\perp} /\left(I_{\mathcal{X}_{2}} M\right)^{\perp}=R_{\mathcal{X}_{1}}\left(M^{*}\right) / R_{\mathcal{X}_{2}}\left(M^{*}\right)
$$

and

$$
\left(R_{\mathcal{X}_{1}} M / R_{\mathcal{X}_{2}} M\right)^{*} \cong\left(R_{\mathcal{X}_{2}} M\right)^{\perp} /\left(R_{\mathcal{X}_{1}} M\right)^{\perp}=I_{\mathcal{X}_{2}}\left(M^{*}\right) / I_{\mathcal{X}_{1}}\left(M^{*}\right) .
$$

Identifying $M$ with its double dual we have $\left(I_{\mathcal{X}}\left(M^{*}\right)\right)^{*}=R_{\mathcal{X}} M$ and $\left(R_{\mathcal{X}}\left(M^{*}\right)\right)^{*}=I_{\mathcal{X}} M$.

Proof. Since $I_{\mathcal{X}} M(K)$ is the sum of the images of all the maps $I_{H}^{K}$ with $H \in \mathcal{X}$ and $H \leq K$ we have

$$
\left(I_{\mathcal{X}} M\right)^{\perp}(K)=\left\{f \in M^{*}(K) \mid f\left(I_{H}^{K}(x)\right)=0 \text { for all } H \in \mathcal{X}, H \leq K \text { and } x \in M(H)\right\} .
$$

Now $f\left(I_{H}^{K}(x)\right)=\left(R_{H}^{K} f\right)(x)$ from the definition of the restriction maps in $M^{*}$, and so the condition is that $R_{H}^{K} f$ is the zero map for all $H \in \mathcal{X}, H \leq K$. This is exactly the condition that $f$ should belong to $R_{\mathcal{X}}\left(M^{*}\right)(K)$, and so $\left(I_{\mathcal{X}} M\right)^{\perp}=R_{\mathcal{X}}\left(M^{*}\right)$. The argument which shows that $\left(R_{\mathcal{X}} M\right)^{\perp}=I_{\mathcal{X}}\left(M^{*}\right)$ is similar, and the remaining assertions are standard deductions. 
Proposition 2.1(b) says that the mappings $\mathcal{X} \mapsto I_{\mathcal{X}} M$ and $\mathcal{X} \mapsto R_{\mathcal{X}} M$ are order preserving and order reversing maps from the lattice of subsets of subgroups of $G$ to the lattice of subfunctors of $M$. Using this we may construct filtrations of $M$ by ordering the conjugacy classes of subgroups of $G$ as $1=H_{1}, H_{2}, \ldots, H_{n}=G$ in such a way that if $H_{i}$ is conjugate to a subgroup of $H_{j}$ then $i \leq j$. Let us write $M_{i}=I_{\left\{H_{1}, \ldots, H_{i}\right\}} M$ and $M^{i}=R_{\left\{H_{1}, \ldots, H_{i}\right\}} M$ with $M_{0}=0$ and $M^{0}=M$. We will call

$$
0=M_{0} \subseteq M_{1} \subseteq \cdots \subseteq M_{n}=M \quad \text { and } \quad M=M^{0} \supseteq M^{1} \supseteq \cdots \supseteq M^{n}=0
$$

the ascending filtration and the descending filtration of $M$ associated to the list $H_{1}, \ldots, H_{n}$. The last results immediately apply to the terms of these filtrations, and we have for all $i$,

- $(M \oplus N)_{i}=M_{i} \oplus N_{i}$,

- $M_{i} / M_{i-1}$ is generated by its value at $H_{i}$,

- if $M$ is projective relative to $\mathcal{Y}$ then so are all the factors $M_{i+r} / M_{i}$,

- $M_{i}+M^{i} \supseteq|G| \cdot M$ and $|G| \cdot\left(M_{i} \cap M^{i}\right)=0$,

- $M_{i}=M^{* i *}$ when $R$ is a field.

We are interested in the factors $M_{i} / M_{i-1}$ and $M^{i-1} / M^{i}$ of these filtrations. Evidently these are associated in some way to the subgroup $H_{i}$, but they depend also on the ordering of the rest of the list of conjugacy classes of subgroups and need not be uniquely determined by $H_{i}$. We will see from the next result that as the position of a particular subgroup $H$ varies between different lists, there is a largest such factor associated to $H$, which occurs when $H$ appears as early as possible in a list, and a smallest such factor which occurs when $H$ appears as late as possible in a list. For lists in which $H$ occurs between the extreme positions, these factors are related by homomorphisms. We will also see in the next results that the values of the factors at $H_{i}$ are in fact determined independently of the position of $H_{i}$ in the list of subgroups, and they are the Brauer quotient and restriction kernel of $M$ at $H_{i}$.

The Brauer quotient of $M$ at $H$ is defined to be

$$
\bar{M}(H)=M(H) / \sum_{J<H} I_{J}^{H}(M(J) .
$$

We also define the restriction kernel of $M$ at $H$, namely

$$
\underline{M}(H)=\bigcap_{J<H} \operatorname{Ker} R_{J}^{H}
$$

These are constructions which appear throughout representation theory. When $R$ is a field the restriction kernel is in fact dual to the Brauer quotient, in that it is the dual of the Brauer quotient of the dual Mackey functor: $\underline{M}(H)=\left(\overline{M^{*}}\right)^{*}$. This is a particular consequence of Proposition 2.2 and the next result, Proposition 2.3. Thus in the context of Mackey functors over a field we may work equally well with either the Brauer quotient or the restriction kernel, although for some reason the Brauer quotient appears more 
frequently than the restriction kernel. In group cohomology the restriction kernel has been termed the essential elements, as studied for example in [1]. A construction with similar formalism (not to do with Mackey functors) underlies the 'kernel intersection theorems' of James in the context of representations of the symmetric and general linear groups, and our descending filtration of $M$ is obtained by an analogous procedure to that which may be used to obtain Specht filtrations of the Young modules as described in [4].

(2.3) PROPOSITION.

(a) Suppose that $\mathcal{X}$ and $\mathcal{Y}$ are sets of subgroups of $G$ with $\mathcal{X} \subseteq \mathcal{Y}$. There is an epimorphism

$$
I_{\mathcal{X} \cup\{H\}} M / I_{\mathcal{X}} M \rightarrow I_{\mathcal{Y} \cup\{H\}} M / I_{\mathcal{Y}} M
$$

and a monomorphism

$$
R_{\mathcal{Y}} M / R_{\mathcal{Y} \cup\{H\}} M \rightarrow R_{\mathcal{X}} M / R_{\mathcal{X} \cup\{H\}} M .
$$

(b) If $\mathcal{X}$ is a set of subgroups of $G$ which contains a conjugate of every subgroup of $H$, but no conjugate of $H$ itself, then

$$
\left(I_{\mathcal{X} \cup\{H\}} M / I_{\mathcal{X}} M\right)(H) \cong \bar{M}(H)
$$

and

$$
\left(R_{\mathcal{X}} M / R_{\mathcal{X} \cup\{H\}} M\right)(H) \cong \underline{M}(H) .
$$

Proof. (a) Since $\mathcal{X} \subseteq \mathcal{Y}$ we have that $I_{\mathcal{X} \cup\{H\}} M$ is a subfunctor of $I_{\mathcal{Y} \cup\{H\}} M$ and $I_{\mathcal{X}} M$ is a subfunctor of $I_{\mathcal{Y}} M$. Thus the composite of inclusion and quotient maps

$$
I_{\mathcal{X} \cup\{H\}} M \rightarrow I_{\mathcal{Y} \cup\{H\}} M \rightarrow I_{\mathcal{Y} \cup\{H\}} M / I_{\mathcal{Y}} M
$$

contains $I_{\mathcal{X}} M$ in the kernel and induces a morphism

$$
I_{\mathcal{X} \cup\{H\}} M / I_{\mathcal{X}} M \rightarrow I_{\mathcal{Y} \cup\{H\}} M / I_{\mathcal{Y}} M .
$$

It is an epimorphism since $I_{\mathcal{Y} \cup\{H\}} M$ is generated by $I_{\mathcal{Y}} M$ and $M(H)$, and $M(H)=$ $I_{\mathcal{X} \cup\{H\}} M(H)$ is in the image of the morphism from $I_{\mathcal{X} \cup\{H\}} M$.

The construction and properties of the morphism

$$
R_{\mathcal{Y}} M / R_{\mathcal{Y} \cup\{H\}} M \rightarrow R_{\mathcal{X}} M / R_{\mathcal{X} \cup\{H\}} M
$$

are established in a dual manner.

(b) Let $\mathcal{X}^{+}$be the conjugacy closure of $\mathcal{X}$. We have from Proposition 2.1

$$
I_{\mathcal{X}} M(H)=I_{\mathcal{X}^{+}} M(H)=\sum_{H \supseteq L \in \mathcal{X}^{+}} I_{L}^{H}(M(L))
$$

and this equals $\sum_{L<H} I_{L}^{H} M(L)$ since only proper subgroups of $H$ appear in $\mathcal{X}^{+}$. Since $I_{\mathcal{X} \cup\{H\}} M(H)=M(H)$, the quotient is $\bar{M}(H)$. This establishes the first formula, and the proof of the second is very similar. 
(2.4) COROLLARY. In any ascending or descending filtration of $M$ corresponding to a list of subgroups $1=H_{1}, H_{2}, \ldots, H_{n}=G$ we have $\left(M_{i} / M_{i-1}\right)\left(H_{i}\right) \cong \bar{M}\left(H_{i}\right)$ and $\left(M^{i-1} / M^{i}\right)\left(H_{i}\right) \cong \underline{M}\left(H_{i}\right)$.

The structure we are observing is connected with the following well-known result.

(2.5) PROPOSITION. Let $f: N \rightarrow M$ be a morphism of Mackey functors.

(a) If the composite morphism $N(H) \rightarrow M(H) \rightarrow \bar{M}(H)$ is surjective for every subgroup $H$ then $f$ is an epimorphism.

(b) If the composite morphism $\underline{N}(H) \rightarrow N(H) \rightarrow M(H)$ is injective for every subgroup $H$ then $f$ is an monomorphism.

Proof. for (a), let $0=M_{0} \subseteq M_{1} \subseteq \cdots$ be an ascending filtration of $M$. We show by induction that $M_{i} \subseteq \operatorname{Im} f$, which is certainly true when $i=0$. Assuming that $M_{i-1} \supseteq \operatorname{Im} f$ we also know that $\left(M_{i} \cap \operatorname{Im} f\right) / M_{i-1}=M_{i} / M_{i-1}$ since this is generated by $\left(M_{i} / M_{i-1}\right)\left(H_{i}\right)$ by $2.1(\mathrm{e})$, which equals $\bar{M}\left(H_{i}\right)$ by $2.3(\mathrm{~b})$ and is covered by $f$. Hence $M_{i} \subseteq \operatorname{Im} f$. The proof of (b) is dual.

\section{The $\Delta$ and $\nabla$ functors}

Whenever $K$ is a group, $U$ is an $R K$-module and $J$ is a subgroup of $K$ we will use the notation $F P_{U}(J)=U^{J}=H^{0}(J, U)$ for the fixed points of $J$ on $U$, and $F Q_{U}(J)=U_{J}=$ $H_{0}(J, U)$ for the fixed quotient (or cofixed points) of $J$ on $U$. Thus $F P_{U}$ and $F Q_{U}$ are the fixed point and fixed quotient Mackey functors.

Let $H$ be a subgroup of $G$ and $U$ an $R\left[N_{G}(H) / H\right]$-module. The functors defined by

$$
\Delta_{H, U}=\left(\operatorname{Inf}_{N(H) / H}^{N(H)} F Q_{U}\right) \uparrow_{N(H)}^{G}
$$

and

$$
\nabla_{H, U}=\left(\operatorname{Inf}_{N(H) / H}^{N(H)} F P_{U}\right) \uparrow_{N(H)}^{G}
$$

in the notation of [13] and [14] will be very important to us, and we now summarize the basic facts about them. The $\nabla_{H, U}$ were first defined in different notation by Lewis [8] and several of the statements we make in this section were familiar to him. They also play an important role in the work of Luca [9], who describes a number of their properties. When $H$ and $K$ are subgroups of $G$ we will write

$$
T(H, K)=\left\{g \in G \mid{ }^{g} H \subseteq K\right\}
$$

for the transporter of $H$ into $K$. By a straightforward modification of the argument in [14, 8.8] we have: 
(3.1) PROPOSITION.

$$
\Delta_{H, U}(K)=\bigoplus_{g \in\left[K \backslash T(H, K) / N_{G}(H)\right]} U_{N_{K^{g}}(H)}
$$

and

$$
\nabla_{H, U}(K)=\bigoplus_{g \in\left[K \backslash T(H, K) / N_{G}(H)\right]} U^{N_{K^{g}}(H)} .
$$

In particular $\Delta_{H, U}(H)=U=\nabla_{H, U}(H)$, and both $\Delta_{H, U}(K)$ and $\nabla_{H, U}(K)$ vanish unless $K$ contains a conjugate of $H$.

In this statement we are using the notation $U_{N_{K^{g}}(H)}$ and $U^{N_{K^{g}}(H)}$ for the fixed quotient and fixed points of $N_{K^{g}}(H)$ on $U$.

Proof. By the formula for an induced functor $[13,4.3]$ we have

$$
\Delta_{H, U}(K)=\bigoplus_{g \in\left[K \backslash G / N_{G}(H)\right]}\left(\operatorname{Inf}_{N(H) / H}^{N(H)} F Q_{U}\right)\left(K^{g} \cap N_{G}(H)\right) .
$$

The terms are zero unless $K^{g} \supseteq H$, i.e. $g \in T(H, K)$. We may write $K^{g} \cap N_{G}(H)=$ $N_{K^{g}}(H)$, and now we have all the ingredients in the formula.

The formula for $\nabla_{H, U}(K)$ is the same, except that we take fixed points instead of fixed quotients.

The main significance of the $\Delta$ and $\nabla$ functors is that they satisfy an adjoint property with respect to the Brauer quotients and restriction kernels.

(3.2) PROPOSITION. For each subgroup $H$ the functor

$$
\operatorname{Mack}_{R}(G) \rightarrow R\left[N_{G}(H) / H\right]-\bmod
$$

specified by $M \mapsto \underline{M}(H)$ has left adjoint $U \mapsto \Delta_{H, U}$. Similarly the functor specified by $M \mapsto \bar{M}(H)$ has right adjoint $U \mapsto \nabla_{H, U}$. The natural isomorphisms $\operatorname{Hom}\left(\Delta_{H, U}, M\right) \rightarrow$ $\operatorname{Hom}(U, \underline{M}(H))$ and $\operatorname{Hom}\left(M, \nabla_{H, U}\right) \rightarrow \operatorname{Hom}(\bar{M}(H), U)$ are given by evaluating morphisms at $H$.

Proof. This appears, for example, in the proof of $[13,8.1]$. We have (in the notation of [13])

$$
\begin{aligned}
\operatorname{Hom}\left(\Delta_{H, U}, M\right) & =\operatorname{Hom}\left(\left(\operatorname{Inf}_{N(H) / H}^{N(H)} F Q_{U}\right) \uparrow_{N(H)}^{G}, M\right) \\
& \cong \operatorname{Hom}\left(\operatorname{Inf}_{N(H) / H}^{N(H)} F Q_{U}, M \downarrow_{N(H)}^{G}\right) \\
& \cong \operatorname{Hom}\left(F Q_{U},\left(M \downarrow_{N(H)}^{G}\right)^{-}\right) \\
& \cong \operatorname{Hom}\left(U,\left(M \downarrow_{N(H)}^{G}\right)^{-}(H / H)\right)
\end{aligned}
$$


passing through a chain of adjunctions. Here

$$
\left(M \downarrow_{N(H)}^{G}\right)^{-}(K / H)=\bigcap_{\substack{J \subseteq K \\ J \not ̄ H}} \operatorname{Ker} R_{J}^{K}
$$

and so its value on the identity subgroup $H / H$ is exactly $\underline{M}(H)$. The precise description of the adjunction isomorphism comes by tracing these isomorphisms through the chain of adjunctions.

The proof for $\nabla_{H, U}$ is similar. When $R$ is a field we may also exploit duality to deduce the properties of $\nabla_{H, U}$ from those of $\Delta_{H, U^{*}}$.

The next corollary may also be expressed as an adjoint property in which $\Delta_{H, U}$ and $\nabla_{H, U}$ are regarded as functors to the full subcategory of Mackey functors which vanish on subgroups which do not contain a conjugate of $H$.

(3.3) COROLLARY. Let $H$ be a subgroup of $G$ and let $M$ be a Mackey functor for which $M(K)=0$ unless $K$ contains a conjugate of $H$. Then every morphism of $R\left[N_{G}(H) / H\right]$-modules $U \rightarrow M(H)$ extends uniquely to a morphism of Mackey functors $\Delta_{H, U} \rightarrow M$, and every morphism of $R\left[N_{G}(H) / H\right]$-modules $M(H) \rightarrow U$ extends uniquely to a morphism of Mackey functors $M \rightarrow \nabla_{H, U}$.

Proof. For such a Mackey functor $M$ we have $\bar{M}(H)=\underline{M}(H)=M(H)$ and so the adjunction isomorphisms become $\operatorname{Hom}\left(\Delta_{H, U}, M\right) \cong \operatorname{Hom}(U, M(H))$ and $\operatorname{Hom}\left(M, \nabla_{H, U}\right) \cong$ $\operatorname{Hom}(M(H), U)$. The statement of the corollary is now another way of saying that these isomorphisms are given by evaluating morphisms at $H$.

From this we obtain the following conclusion:

(3.4) COROLLARY. $\Delta_{H, U}$ is generated by its value at $H$, and $\nabla_{H, U}$ is cogenerated by its value at $H$. If $U$ is an indecomposable module then the Mackey functors $\Delta_{H, U}$ and $\nabla_{H, U}$ are indecomposable.

Proof. Suppose $f: M \rightarrow \Delta_{H, U}$ is a morphism for which $M(H) \rightarrow \Delta_{H, U}(H)=U$ is surjective, and consider the quotient morphism $\Delta_{H, U} \rightarrow \Delta_{H, U} / f(M)$. This morphism is zero on $U$ and by 3.3 it is the unique extension of its value on $U$. Since zero is such an extension we obtain that the quotient map is zero, and hence $f(M)=\Delta_{H, U}$. The proof for $\nabla_{H, U}$ is dual.

Now if $U$ is indecomposable and $\Delta_{H, U}=M_{1} \oplus M_{2}$ for some Mackey functors $M_{1}$ and $M_{2}$ then $\Delta_{H, U}(H)=U=M_{1}(H) \oplus M_{2}(H)$ so one of the terms on the right - say $M_{2}(H)$ - is zero. Since $\Delta_{H, U}$ is generated by its value at $H$, so is $M_{2}$, and hence $M_{2}=0$. Again the proof for $\nabla_{H, U}$ is similar. 
We have seen in Proposition 3.2 that the functor $\Delta_{H, U}$ is associated by means of an adjoint property with the restriction kernel, and $\nabla_{H, U}$ is associated with the Brauer quotient. In the next result it is a little surprising that these associations are reversed, and $\Delta$ is used in combination with a Brauer quotient while $\nabla$ is used in combination with a restriction kernel. We comment further on this after Corollary 3.6.

(3.5) COROLLARY. Consider the ascending and descending filtrations of a Mackey functor $M$ associated to a list $1=H_{1}, H_{2}, \ldots, H_{n}=G$ of subgroups of $G$ taken up to conjugacy, in non-decreasing order. For each $i$ there are epimorphisms

$$
\Delta_{H_{i}, \bar{M}\left(H_{i}\right)} \rightarrow M_{i} / M_{i-1}
$$

and there are monomorphisms

$$
M^{i-1} / M^{i} \rightarrow \nabla_{H_{i}, \underline{M}}\left(H_{i}\right)
$$

Proof. By 2.4 we have that $M_{i} / M_{i-1}\left(H_{i}\right)=\bar{M}\left(H_{i}\right)$ is the Brauer quotient at $H_{i}$ and $M^{i-1} / M^{i}\left(H_{i}\right)=\underline{M}\left(H_{i}\right)$ is the restriction kernel. Thus the identity morphisms on these modules correspond via the adjunctions implicit in 3.3 to the claimed morphisms of Mackey functors in the present statement, since $M_{i} / M_{i-1}$ and $M^{i-1} / M^{i}$ vanish on groups which do not contain a conjugate of $H_{i}$ as a subgroup. The first morphism is an epimorphism because $M_{i} / M_{i-1}$ is generated by its value at $H_{i}$, and the second is a monomorphism because $M^{i-1} / M^{i}$ is cogenerated by its value at $H_{i}$.

(3.6) COROLLARY. Let $M$ be a Mackey functor defined over a field $R$. For each subgroup $K$ we have

$$
\operatorname{dim} M(K) \leq \sum_{\substack{H \leq G \\ \text { up to conjugacy }}} \operatorname{dim} \Delta_{H, \bar{M}(H)}(K)
$$

and

$$
\operatorname{dim} M(K) \leq \sum_{\substack{H \leq G \\ \text { up to conjugacy }}} \operatorname{dim} \nabla_{H, \underline{M}(H)}(K)
$$

Proof. We take a list $1=H_{1}, H_{2}, \ldots, H_{n}=G$ of subgroups of $G$ taken up to conjugacy, in non-decreasing order. Now $\operatorname{dim} M(K)=\sum_{i=1}^{n} \operatorname{dim}\left(M_{i} / M_{i-1}\right)(K)$ and each of these dimensions in the sum is bounded by $\operatorname{dim} \Delta_{H_{i}, \bar{M}\left(H_{i}\right)}(K)$, by 3.5 . The second inequality is proved similarly. 
These inequalities bound the dimension of $M(K)$ solely in terms of the Brauer quotients of $M$, or the restriction kernels of $M$, since the dimensions of $\Delta$ and $\nabla$ may be deduced from the explicit formulas for them given in Proposition 3.1. The bounds are best possible in general, and indeed the rest of this paper is devoted to a study of Mackey functors for which equality holds. As a particular example, it will be proved that the first inequality is an equality whenever $M$ is projective, from which we can deduce by duality that the second inequality is an equality whenever $M$ is injective.

We point out a difference between Corollary 3.6 and a confusingly similar construction which underlies the twin functor of Thévenaz [11]. By Proposition 3.2 the identity morphism $\bar{M}(H) \rightarrow \bar{M}(H)=U$ corresponds to a morphism of Mackey functors $M \rightarrow \nabla_{H, \bar{M}(H)}$, and also the identity morphism $U=\underline{M}(H) \rightarrow \underline{M}(H)$ corresponds to a morphism $\Delta_{H, \underline{M}(H)} \rightarrow M$. We may take the first of these morphisms to be the components in a morphism

$$
M \rightarrow \prod_{\substack{H \subseteq G \\ \text { up to conjugacy }}} \nabla_{H, \bar{M}(H)}
$$

and at first sight it appears that we may be able to deduce something like the inequalities of Corollary 3.6 from this. The target is in fact the twin functor of $M$ as studied by Thévenaz [11] (see also $[13,9.3]$ ). It is shown there that when $|G|$ is invertible in $R$ this morphism is an isomorphism. When $|G|$ is not invertible it need not be even an monomorphism, and so an inequality does not immediately follow. On closer examination the $\nabla$ inequality of 3.6 involves the restriction kernel, whereas the twin functor is expressed in terms of the Brauer quotient, so the ingredients in these constructions are rather different. 


\section{Induction, restriction and inflation of $\Delta_{H, U}$ and $\nabla_{H, U}$}

In this section we consider the effect of some standard operations on the Mackey functors $\Delta_{H, U}$. In order to specify the group for which these are Mackey functors we will quite often write this group as a superscript, so that for example $\Delta_{H, U}^{K}$ indicates that this is an object of $\operatorname{Mack}_{R}(K)$, where $H \subseteq K$. The results in this section are rather formal and at times uninspiring, but they do provide crucial simplifications in our arguments later on. As always, similar results hold for both the $\Delta_{H, U}$ and $\nabla_{H, U}$. We will work mainly with the $\Delta_{H, U}$ and leave it to the reader to formulate and prove the results for $\nabla_{H, U}$ when necessary.

(4.1) LEMMA. Suppose that $H \subseteq J \subseteq G$ and that $U$ is an $R\left[N_{J}(H) / H\right]$-module. Then $\Delta_{H, U}^{J} \uparrow_{J}^{G} \cong \Delta_{H, U \uparrow_{N_{J}(H)}^{N_{G}(H)}}^{G}$.

Proof. We are abusing notation by writing $U \uparrow_{N_{J}(H)}^{N_{G}(H)}$ instead of $U \uparrow_{N_{J}(H) / H}^{N_{G}(H) / H}$, and we will repeatedly do this. We have

$$
\begin{aligned}
\Delta_{H, U}^{J} \uparrow G & \cong\left(\operatorname{Inf}_{H}^{N_{J}(H)} F Q_{U}\right) \uparrow_{N_{J}(H)}^{J} \uparrow_{J}^{G} \\
& \cong\left(\operatorname{Inf}_{H}^{N_{J}(H)} F Q_{U}\right) \uparrow_{N_{J}(H)}^{N_{G}(H)} \uparrow_{N_{G}(H)}^{G} \\
& \cong\left(\operatorname{Inf}_{H}^{N_{G}(H)}\left(\left(F Q_{U}\right) \uparrow_{N_{J}(H) / H}^{N_{G}(H) / H}\right) \uparrow_{N_{G}(H)}^{G}\right. \\
& \cong\left(\operatorname { I n f } _ { H } ^ { N _ { G } ( H ) } \left(F Q_{\left.U \uparrow_{N_{J}(H) / H}^{N_{G}(H) / H}\right)} \uparrow_{N_{G}(H)}^{G} \quad \text { by }[14,5.2]\right.\right. \\
& \cong \Delta_{H, U_{N_{J}(H)}^{N_{G}(H)}}^{G} .
\end{aligned}
$$

The third isomorphism comes from the definitions of inflation and induction.

\section{(4.2) COROLLARY.}

(a) If $N_{G}(H) \subseteq J$ then $\Delta_{H, U}^{J} \uparrow{ }_{J}^{G} \cong \Delta_{H, U}^{G}$ and $\nabla_{H, U}^{J} \uparrow_{J}^{G} \cong \nabla_{H, U}^{G}$.

(b) Suppose that $R$ is a complete p-local ring. Then every Mackey functor $\Delta_{H, U}^{G}$ with $U$ an indecomposable p-permutation module arises as a summand of a functor $\Delta_{H, R}^{K} \uparrow_{K}^{G}$ where $H \triangleleft K$ and $H / K$ is a $p$-group.

(c) Suppose that $R$ is a complete p-local ring and that $H \subseteq J \subseteq G$. If $U$ is an indecomposable p-permutation $R\left[N_{J}(H) / H\right]$-module then $\Delta_{H, U}^{J} \uparrow_{J}^{G}$ is a direct sum of indecomposable Mackey functors $\Delta_{H, W}$ where $W$ is a p-permutation $R\left[N_{G}(H) / H\right]$ module with vertex vx $W \subseteq_{N_{G}(H) / H} \mathrm{vx} U$.

Proof. (a) is a particular case of Lemma 4.1, but it is also immediate from the definitions by transitivity of induction. 
(b) Since $U$ is a $p$-permutation module for $R\left[N_{G}(H) / J\right]$ it is a summand of a module $R \uparrow_{K}^{N_{G}(H)}$ for some subgroup $K$ with $H \triangleleft K$ and $K / H$ a $p$-group. Thus $\Delta_{H, U}^{G}$ is a summand of $\Delta_{H, R \uparrow_{K}^{N}}^{N_{G}(H)}$, which is isomorphic to $\Delta_{H, R}^{K} \uparrow_{K}^{G}$ by Lemma 4.1.

(c) Here $\Delta_{H, U}^{J} \uparrow_{J}^{G} \cong \Delta_{H, U \uparrow_{N_{J}(H)}^{N_{G}(H)}}^{G}$ is the direct sum of Mackey functors $\Delta_{H, W}$ where $W$ ranges over the indecomposable summands of $U \uparrow_{N_{J}(H)}^{N_{G}(H)}$. These modules have the stated form, and the functors $\Delta_{H, W}$ are indecomposable by 3.4 .

(4.3) LEMMA. Let $L$ be a subgroup of $G$. Then

$$
\Delta_{H, U}^{G} \downarrow_{L}^{G} \cong \bigoplus_{x \in\left[L \backslash T(H, L) / N_{G}(H)\right]} x\left(\Delta_{H, U}^{L^{x}}\right)
$$

where on the right we interpret $U$ as a module for $N_{L^{x}}(H) / H$ by restriction from $N_{G}(H) / H$. It follows that when $R$ is a complete $p$-local ring and $U$ is an indecomposable p-permutation module then $\Delta_{H, U}^{G} \downarrow_{L}^{G}$ is a direct sum of indecomposable functors ${ }^{x}\left(\Delta_{H, W}^{L^{x}}\right) \cong \Delta_{x_{H},{ }^{x} W}^{L}$ where ${ }^{x} W$ is a $p$-permutation $R\left[N_{L}\left({ }^{x} H\right) /{ }^{x} H\right]$-module with vx ${ }^{x} W \subseteq{ }_{N_{L}\left({ }^{x} H\right) /{ }^{x} H}$ vx ${ }^{x} U$.

Proof. We can write $\Delta_{H, U}^{G}=\Delta_{H, U}^{N_{G}(H)} \uparrow_{N_{G}(H)}^{G}$ and so

$$
\begin{aligned}
\Delta_{H, U}^{G} \downarrow_{L}^{G} & =\Delta_{H, U}^{N_{G}(H)} \uparrow_{N_{G}(H)}^{G} \downarrow_{L}^{G} \\
& =\bigoplus_{x \in\left[L \backslash G / N_{G}(H)\right]}^{x}\left(\Delta_{H, U}^{N_{G}(H)} \downarrow_{L^{x} \cap N_{G}(H)}^{N_{G}(H)} \uparrow_{L^{x} \cap N_{G}(H)}^{L^{x}}\right) .
\end{aligned}
$$

Now since $\Delta_{H, U}^{N_{G}(H)}=\operatorname{Inf}_{N_{G}(H) / H}^{N_{G}(H)} F Q_{U}$ the restriction $\Delta_{H, U}^{N_{G}(H)} \downarrow_{L^{x} \cap N_{G}(H)}^{N_{G}(H)}$ is zero unless $L^{x} \cap N_{G}(H) \supseteq H$, that is, $x \in T(H, L)$ and then $\Delta_{H, U}^{N_{G}(H)} \downarrow_{L^{x} \cap N_{G}(H)}^{N_{G}(H)}=\Delta_{H, U}^{L^{x} \cap N_{G}(H)}$. Thus

$$
\begin{aligned}
& \Delta_{H, U}^{G} \downarrow_{L}^{G} \cong \bigoplus_{x \in\left[L \backslash T(H, L) / N_{G}(H)\right]} x\left(\Delta_{H, U}^{L^{x} \cap N_{G}(H)} \uparrow_{L^{x} \cap N_{G}(H)}^{L^{x}}\right) \\
& \cong \bigoplus_{x \in\left[L \backslash T(H, L) / N_{G}(H)\right]} x\left(\Delta_{H, U}^{L^{x}}\right)
\end{aligned}
$$

The last statements are clear.

(4.4) COROLLARY. If $N_{G}(H) \subseteq L$ then $\Delta_{H, U}^{L}$ is isomorphic to the subfunctor of $\Delta_{H, U}^{G} \downarrow_{L}^{G}$ generated by the value of this Mackey functor at $H$, and this is a direct summand of $\Delta_{H, U}^{G} \downarrow_{L}^{G}$.

Proof. We have $\Delta_{H, U}^{G} \downarrow_{L}^{G} \cong \bigoplus_{x \in\left[L \backslash T(H, L) / N_{G}(H)\right]}{ }^{x}\left(\Delta_{H, U}^{L^{x}}\right)$ and the summand corresponding to $x=1$ is isomorphic to $\Delta_{H, U}^{L}$. Since $\Delta_{H, U}^{L}(H) \cong \Delta_{H, U}^{G}(H) \cong U$ it follows that all the other summands in the direct sum are zero at $H$. Hence the subfunctor of $\Delta_{H, U}^{G} \downarrow_{L}^{G}$ generated by the value at $H$ is precisely the summand $\Delta_{H, U}^{L}$, since this summand is itself generated by its value at $H$ (and furthermore we see that this summand is a uniquely determined subfunctor). 
In the same spirit we note the following particular identities.

(4.5) COROLLARY. If $H \triangleleft J \subseteq G$ and $L \subseteq G$ then

$$
\begin{aligned}
\left(\Delta_{H, R \uparrow_{J}^{N} G(H)}^{G}\right) \downarrow_{L}^{G} & =\left(\Delta_{H, R}^{J} \uparrow_{J}^{G}\right) \downarrow_{L}^{G} \\
& =\bigoplus_{x \in[L \backslash T(H, L) / J]} \Delta_{x_{H, R} \uparrow_{L \cap x_{J}}^{N_{L}\left(x_{H}\right)}}^{L}\left(\Delta_{x \in[L \backslash T(H, L) / J]}^{L \cap^{x} J}\right) \uparrow_{L \cap \cap^{x} J}^{L} \\
& =\bigoplus_{x \in R} .
\end{aligned}
$$

The following result is really present in $[14,11.4]$, but it also follows in a straightforward way from our results about induction and restriction.

(4.6) COROLLARY. Suppose that $R$ is a complete p-local ring and that $U$ is an indecomposable $R\left[N_{G}(H) / H\right]$-module with vertex $K / H$ where $H \triangleleft K$. Then $K$ is a vertex of $\Delta_{H, U}$.

Proof. If $U$ is a summand of $R \uparrow_{K}^{N_{G}(H)}$ then $\Delta_{H, U}$ is a summand of $\Delta_{H, R \uparrow_{K}^{N_{G}(H)}} \cong$ $\Delta_{H, R}^{K} \uparrow_{K}^{G}$. This shows that $K$ contains a vertex of $\Delta_{H, U}$. On the other hand, suppose that $\Delta_{H, U}$ is a summand of $\Delta_{H, U} \downarrow_{L}^{G} \uparrow_{L}^{G}$ where $L \subseteq K$. We can write this as

$$
\bigoplus_{x \in\left[L \backslash T(H, L) / N_{G}(H)\right]}\left(\Delta_{x_{H},{ }^{x} U}^{L}\right) \uparrow_{L}^{G} \cong \bigoplus_{x \in\left[L \backslash T(H, L) / N_{G}(H)\right]} \Delta_{x^{\prime} H,{ }^{x} \uparrow_{L}^{N_{G}(H)}}^{G} .
$$

Now $U=\Delta_{H, U}(H)$ is a summand of the previous direct sum, evaluated at $H$, which is

$$
\bigoplus_{x \in\left[L \backslash T(H, L) / N_{G}(H)\right]}{ }^{x} U \uparrow_{L}^{N_{G}(H)}
$$

and since all summands are induced from $L$ we deduce that $L$ must contain a vertex of $U$. We deduce that $L=K$ and so $K$ is a vertex of $\Delta_{H, U}$.

The following lemma is immediate from the definitions.

(4.7) LEMMA. Let $N \triangleleft G, N \subseteq H \subseteq G$, and let $U$ be an $N_{G}(H) / H$ module. Then $\operatorname{Inf}_{G / N}^{G}\left(\Delta_{H / N, U}^{G / N}\right) \cong \Delta_{H, U}$ and $\operatorname{Inf}_{G / N}^{G}\left(\nabla_{H / N, U}^{G / N}\right) \cong \nabla_{H, U}$.

As a consequence of what we have just done we see that the Mackey functors $\Delta_{H, U}$ where $U$ is a $p$-permutation module are precisely what we obtain by starting with the Mackey functor $F Q_{R}$ and repeatedly applying inflation and induction, and taking direct sums and direct summands. 
To conclude this section we define one of the main objects of our study. This is the category $\mathcal{D}$ which is the full subcategory of the category $\operatorname{Mack}_{R}(G)$ of Mackey functors whose objects are the functors with a finite filtration

$$
0=F_{0} M \subseteq F_{1} M \subseteq \cdots \subseteq F_{n} M=M
$$

in which for every $i$ the factor $F_{i} M / F_{i-1} M$ is isomorphic to $\Delta_{H_{i}, U_{i}}$ for some subgroup $H_{i}$ and finitely-generated $p$-permutation $R\left[N_{G}\left(H_{i}\right) / H_{i}\right]$-module $U_{i}$. We will refer to objects in $\mathcal{D}$ as 'functors with a $\Delta$-filtration'. As $G$ varies, the categories $\mathcal{D}$ behave remarkably well with respect to induction and restriction.

(4.8) COROLLARY. Let $H$ be a subgroup of $G$. Then restriction and induction between $G$ and $H$ preserve the categories $\mathcal{D}$. More specifically, if we denote by $\mathcal{D}^{H}$ and $\mathcal{D}^{G}$ the categories $\mathcal{D}$ for each of these groups then if $M \in \mathcal{D}^{G}$ we have $M \downarrow_{H}^{G} \in \mathcal{D}^{H}$ and if $M \in \mathcal{D}^{H}$ we have $M \uparrow_{H}^{G} \in \mathcal{D}^{H}$.

\section{Vanishing of Ext-groups}

From this point on we will assume all the time that $R$ is either a field of characteristic $p$ or a complete discrete valuation ring with residue field of characteristic $p$ - what we have been calling a complete $p$-local ring. We have defined the functors $\Delta_{H, U}$ and $\nabla_{H, U}$ for arbitrary $R\left[N_{G}(H) / H\right]$-modules $U$, but now we will restrict these modules to be ppermutation modules, that is, summands of permutation $R\left[N_{G}(H) / H\right]$-modules. The first result in this section will be the key tool which makes our results on stratifications work. Extensions of Mackey functors are the same thing as extensions of modules for the Mackey algebra $\mu_{R}(G)$. To simplify the notation as much as possible in the proof we will sometimes write $\operatorname{Ext}_{\mu(G)}$ or just Ext instead of $\operatorname{Ext}_{\mu_{R}(G)}^{1}$.

(5.1) THEOREM. Suppose that $R$ is a complete p-local ring. Let $H$ and $K$ be subgroups of $G$ and let $U$ and $W$ be p-permutation modules for $R\left[N_{G}(H) / H\right]$ and $R\left[N_{G}(K) / K\right]$ respectively. Then

(a) $\operatorname{Ext}_{\mu_{R}(G)}^{1}\left(\Delta_{H, U}, \Delta_{K, W}\right)=0$ unless $H>_{G} K$.

(b) $\operatorname{Ext}_{\mu_{R}(G)}^{1}\left(\Delta_{H, U}, \nabla_{K, W}\right)=0$ unless $H={ }_{G} K$.

(c) $\operatorname{Ext}_{\mu_{R}(G)}^{1}\left(\nabla_{H, U}, \nabla_{K, W}\right)=0$ unless $H<_{G} K$.

Proof. (a) There will be several reductions. The first is that we may assume that $H$ and $K$ are $p$-subgroups of $G$, which by $[14,9.14]$ is the same thing as requiring that $\Delta_{H, U}$ and $\Delta_{K, W}$ lie in $\operatorname{Mack}_{R}(G, 1)$. We recall from [14] that the expression $1=$ $\sum_{p-\text { perfect } J \leq G} f_{J}$ as a sum of primitive orthogonal idempotents in the Burnside ring 
$R \otimes_{\mathbb{Z}} B(G)$ gives rise to a decomposition $M=\bigoplus_{p-\text { perfect } J \leq G} f_{J} M$ of every Mackey functor $M$ where $f_{J} M \in \operatorname{Mack}_{R}(G, J)$. Summands of different $f_{J} M$ lie in different blocks of Mackey functors, and so to have a non-zero Ext group between indecomposable $\Delta_{H, U}$ and $\Delta_{K, W}$ these Mackey functors must belong to the same category $\operatorname{Mack}_{R}(G, J)$. This is the same as requiring that $O^{p}(H)$ and $O^{p}(K)$ are both conjugate to $J$, and in our notation we will assume that they are equal to $J$. Now there is an equivalence of categories

$$
\left(\operatorname{Inf}_{N(J) / J}^{N(J)}-\right) \uparrow_{N(J)}^{G}: \operatorname{Mack}_{R}(N(J) / J, 1) \rightarrow \operatorname{Mack}_{R}(G, J)
$$

(see $[14,10.1]$ ) and by Lemmas 4.7 and 4.1 the functors $\Delta_{H / J, U}^{N_{G}(J) / J}$ are sent to the functors $\Delta_{H, U}^{G}$ under this equivalence. In verifying this we use the fact that if $J$ is $p$-perfect and $H / J$ is a $p$-group then $N_{G}(H) \subseteq N_{G}(J)$. Thus to determine whether or not $\operatorname{Ext}_{\mu_{R}(G)}^{1}\left(\Delta_{H, U}, \Delta_{K, W}\right)$ is zero it suffices to determine whether or not $\operatorname{Ext}_{\mu_{R}\left(N_{G}(J) / J\right)}^{1}\left(\Delta_{H / J, U}^{N_{G}(J) / J}, \Delta_{K / J, W}^{N_{G}(J) / J}\right)$ is zero, and now $H / J$ and $K / J$ are $p$-groups. In view of all this we may henceforth assume $H$ and $K$ are $p$-subgroups.

By 4.2 every $\Delta_{H, U}$ is a direct summand of a functor $\Delta_{H, R \uparrow_{F}^{N_{G}(H)}}^{G} \cong \Delta_{H, R}^{F} \uparrow_{F}^{G}$ where $H \triangleleft F$ and $F$ is a $p$-group, and similarly $\Delta_{K, W}$ is a summand of a functor $\Delta_{K, R \uparrow_{J}^{N_{G}(K)}}^{G} \cong$ $\Delta_{K, R}^{J} \uparrow_{J}^{G}$ where $K \triangleleft J$ and $J$ is a $p$-group. It suffices to show that $\operatorname{Ext}\left(\Delta_{H, R}^{F} \uparrow_{F}^{G}, \Delta_{K, R}^{J} \uparrow_{J}^{G}\right)=$ 0 in this situation, or in other words we may assume that $U=R \uparrow_{F}^{N_{G}(H)}$ and $W=R \uparrow_{J}^{N_{G}(K)}$ where $F$ and $J$ are $p$-groups.

It also suffices to prove the result when $R$ is a field. This is because if $R$ is a complete discrete valuation ring with maximal ideal $(\pi)$ then $\Delta_{K, W} / \pi \Delta_{K, W} \cong \Delta_{K, W / \pi W}$ since factoring out $\pi$ commutes with the operations of induction, inflation and taking fixed quotients of the trivial module. Now the exact sequence

$$
\Delta_{K, W} \stackrel{\pi}{\rightarrow} \Delta_{K, W} \rightarrow \Delta_{K, W / \pi W}
$$

gives an exact sequence

$$
\begin{aligned}
\operatorname{Ext}\left(\Delta_{H, U}, \Delta_{K, W}\right) \stackrel{\pi}{\rightarrow} \operatorname{Ext}\left(\Delta_{H, U}, \Delta_{K, W}\right) \rightarrow \operatorname{Ext}\left(\Delta_{H, U}, \Delta_{K, W / \pi W}\right) \\
\\
\cong \operatorname{Ext}\left(\Delta_{H, U / \pi U}, \Delta_{K, W / \pi W}\right)
\end{aligned}
$$

which embeds

$$
\operatorname{Ext}\left(\Delta_{H, U}, \Delta_{K, W}\right) / \pi \operatorname{Ext}\left(\Delta_{H, U}, \Delta_{K, W}\right)
$$

as a subgroup of $\operatorname{Ext}\left(\Delta_{H, U / \pi U}, \Delta_{K, W / \pi W}\right)$. If we can prove that the latter group is zero then it follows that $\operatorname{Ext}\left(\Delta_{H, U}, \Delta_{K, W}\right)=0$ by Nakayama's lemma. This justifies our claim that it suffices to assume $R$ is a field.

In what follows we will use various change of rings isomorphisms of the type

$$
\operatorname{Ext}_{\mu(G)}\left(M \uparrow_{F}^{G}, N\right) \cong \operatorname{Ext}_{\mu(F)}\left(M, N \downarrow_{F}^{G}\right) .
$$


These are valid because the induction functor is both the left and the right adjoint of restriction [13] so that the conditions in e.g. [7, IV.12] are satisfied. We have

$$
\begin{aligned}
\operatorname{Ext}_{\mu(G)}\left(\Delta_{H, R}^{F} \uparrow_{F}^{G}, \Delta_{K, R}^{J} \uparrow G\right) & \cong \operatorname{Ext}_{\mu(F)}\left(\Delta_{H, R}^{F}, \Delta_{K, R}^{J} \uparrow_{J}^{G} \downarrow_{F}^{G}\right) \\
& \cong \bigoplus_{g \in[F \backslash T(K, F) / J]} \operatorname{Ext}_{\mu(F)}\left(\Delta_{H, R}^{F}, \Delta_{g_{K}, R}^{F \cap{ }^{g} \uparrow_{F}^{F}{ }^{g} J}\right) \quad \text { by } 4.5 \\
& \cong \bigoplus_{g \in[F \backslash T(K, F) / J]} \operatorname{Ext}_{\mu\left(F \cap{ }^{g} J\right)}\left(\Delta_{H, R}^{F} \downarrow_{F \cap{ }^{g} J}^{F}, \Delta_{g_{K}, R}^{F \cap{ }^{g} J}\right) \\
& \cong \bigoplus_{g \in[F \backslash T(K, F) \cap T(H, J)-1 / J]} \operatorname{Ext}_{\mu\left(F \cap{ }^{g} J\right)}\left(\Delta_{H, R}^{F \cap{ }^{g} J}, \Delta_{g_{K}, R}^{F \cap{ }^{g} J}\right)
\end{aligned}
$$

using 4.3 in the last isomorphism and the fact that $H \triangleleft F$. Writing $L=F \cap{ }^{g} J$, the various summands all have the form $\operatorname{Ext}_{\mu(L)}\left(\operatorname{Inf}_{L / H}^{L} F Q_{R}, \operatorname{Inf}_{L /{ }^{g} K}^{L} F Q_{R}\right)$ where $L$ is a $p$-group in which both $H$ and ${ }^{g} K$ are normal. We will show that all Ext groups of this kind are zero unless $H$ properly contains ${ }^{g} K$.

As a functor on $L /{ }^{g} K, F Q_{R}$ has simple socle $S_{L /{ }^{g} K, R}$ (by the dual result to [14, 15.3], or by $[14,12.4]$ from which it may also be deduced). Hence $\operatorname{Inf}_{L /{ }^{g} K}^{L} F Q_{R}$ has simple socle $S_{L, R}$ and we have an injective presentation

$$
0 \rightarrow \operatorname{Inf}_{L / g_{K}}^{L} F Q_{R} \rightarrow B^{L *} \rightarrow X \rightarrow 0
$$

where $B^{L *}$ is the dual of the Burnside ring functor for the group $L$ and $X$ is defined as the cokernel in this sequence. The fact that $B^{L *}$ is the injective hull of $S_{L, R}$ as a Mackey functor for $L$ follows from [14, 8.9(ii)], and we use this to compute Ext via the exact sequence

$$
\begin{aligned}
\operatorname{Hom}\left(\operatorname{Inf}_{L / H}^{L} F Q_{R}, B^{L *}\right) \rightarrow & \operatorname{Hom}\left(\operatorname{Inf}_{L / H}^{L} F Q_{R}, X\right) \\
& \rightarrow \operatorname{Ext}\left(\operatorname{Inf}_{L / H}^{L} F Q_{R}, \operatorname{Inf}_{L / g_{K}}^{L} F Q_{R}\right) \rightarrow 0
\end{aligned}
$$

By adjointness (Proposition 3.2) this is isomorphic to an exact sequence

$$
\operatorname{Hom}\left(R, \underline{B^{L *}}(H)\right) \rightarrow \operatorname{Hom}(R, \underline{X}(H)) \rightarrow \operatorname{Ext}\left(\operatorname{Inf}_{L / H}^{L} F Q_{R}, \operatorname{Inf}_{L / g_{K}}^{L} F Q_{R}\right) \rightarrow 0 .
$$

If $H \nsupseteq{ }^{g} K$ then $\left(\operatorname{Inf}_{L /{ }^{g} K}^{L} F Q_{R}\right)(A)=0$ for all subgroups $A$ of $H$ so from $(*)$ we obtain that $\underline{B^{L *}}(H) \rightarrow \underline{X}(H)$ is an isomorphism and homomorphisms to $\underline{X}(H)$ factor through $\underline{B^{L *}}(H)$. If $H={ }^{g} K$ then consider $(*)$ again evaluated at $H$ :

$$
0 \rightarrow \operatorname{Inf}_{L / H}^{L} F Q_{R}(H)=R \stackrel{\alpha}{\longrightarrow} B^{L *}(H) \stackrel{\beta}{\longrightarrow} X(H) \rightarrow 0
$$

We claim that $\underline{B^{L *}}(H)=R$. This is equivalent to the statement that $\overline{B^{L}}(H)=R$, by 2.2 and 2.3 as pointed out there. In $B^{L}(H)$ all $H$-sets except the one-point set are induced from proper subgroups, so that the Brauer quotient has dimension 1. Also $R=\operatorname{Im}(\alpha) \subseteq \underline{B^{L *}}(H)$ 
since the domain of $\alpha$ restricts to 0 on all proper subgroups of $H$ so $\operatorname{Im}(\alpha)=\underline{B^{L *}}(H)$ since $R$ is currently assumed to be a field. We show that $\underline{X}(H)=0$. If $0 \neq x \in X(H)$ we can write $x=\beta(y)$ with $y \notin \operatorname{Im}(\alpha)$. Thus there exists $H_{1} \subset H$ with $H_{1} \neq H$ and $R_{H_{1}}^{H}(y) \neq 0$. Now since $\operatorname{Inf}_{L / H}^{L} F Q_{R}\left(H_{1}\right)=0$ we have $R_{H_{1}}^{H}(x) \neq 0$. We conclude that $\underline{X}(H)=0$. By examining the sequence which computes Ext we deduce that this Ext group is zero when $H={ }^{g} K$, and so in all cases $\operatorname{Ext}\left(\operatorname{Inf}_{L / H}^{L} F Q_{R}, \operatorname{Inf}_{L /{ }^{g} K}^{L} F Q_{R}\right)=0$ if $H \ngtr{ }^{g} K$.

(b) We proceed as in part (a), but with $F P_{R}$ instead of $F Q_{R}$ in the second variable, and reduce to considering $\operatorname{Ext}_{\mu(L)}\left(\operatorname{Inf}_{L / H}^{L} F Q_{R}, \operatorname{Inf}_{L /{ }^{g} K}^{L} F P_{R}\right)$. Now as a functor on $L /{ }^{g} K$, $F P_{R}$ has simple socle $S_{g_{K} /{ }^{g} K, R}$, so $\operatorname{Inf}_{L / g_{K}}^{L} F P_{R}$ has simple socle $S_{g_{K}, R}$. We take an injective presentation

$$
0 \rightarrow \operatorname{Inf}_{L / g_{K}}^{L} F P_{R} \rightarrow I_{g_{K}, R} \rightarrow Y \rightarrow 0
$$

giving an exact sequence

$$
\begin{aligned}
\operatorname{Hom}\left(\operatorname{Inf}_{L / H}^{L} F Q_{R}, I_{g_{K}, R}\right) \rightarrow & \operatorname{Hom}\left(\operatorname{Inf}_{L / H}^{L} F Q_{R}, Y\right) \\
& \rightarrow \operatorname{Ext}\left(\operatorname{Inf}_{L / H}^{L} F Q_{R}, \operatorname{Inf}_{L / g_{K}}^{L} F P_{R}\right) \rightarrow 0
\end{aligned}
$$

which by adjointness is isomorphic to

$$
\operatorname{Hom}\left(R, \underline{I_{g_{K}, R}}(H)\right) \rightarrow \operatorname{Hom}(R, \underline{Y}(H)) \rightarrow \operatorname{Ext}\left(\operatorname{Inf}_{L / H}^{L} F Q_{R}, \operatorname{Inf}_{L /{ }^{g} K}^{L} F P_{R}\right) \rightarrow 0
$$

If $H \nsupseteq{ }^{g} K$ then $\left(\operatorname{Inf}_{L / g_{K}}^{L} F P_{R}\right)(A)=0$ for all subgroups $A$ of $H$ so from (**) we obtain that $I_{g_{K}, R}(H) \rightarrow \underline{Y}(H)$ is an isomorphism and homomorphisms to $\underline{Y}(H)$ factor through $\underline{I_{g_{K, R}}} \overline{(H)}$. This shows that if $H \nsupseteq{ }^{g} K$ then

$$
\operatorname{Ext}\left(\operatorname{Inf}_{L / H}^{L} F Q_{R}, \operatorname{Inf}_{L / g_{K}}^{L} F P_{R}\right)=0
$$

We may proceed further and deduce immediately by duality that when $H \nsupseteq{ }^{g} K$ then

$$
\operatorname{Ext}\left(\left(\operatorname{Inf}_{L / g_{K}}^{L} F P_{R}\right)^{*},\left(\operatorname{Inf}_{L / H}^{L} F Q_{R}\right)^{*}\right)=0
$$

or in other words $\operatorname{Ext}\left(\operatorname{Inf}_{L / g_{K}}^{L} F Q_{R}, \operatorname{Inf}_{L / H}^{L} F P_{R}\right)=0$ since $F P_{R}$ and $F Q_{R}$ are dual. Interchanging the roles of $H$ and ${ }^{g} K$ we obtain that when ${ }^{g} K \nsupseteq H$ then

$$
\operatorname{Ext}\left(\operatorname{Inf}_{L / H}^{L} F Q_{R}, \operatorname{Inf}_{L / g_{K}}^{L} F P_{R}\right)=0
$$

Putting everything together we have that $\operatorname{Ext}\left(\operatorname{Inf}_{L / H}^{L} F Q_{R}, \operatorname{Inf}_{L /{ }^{g} K}^{L} F P_{R}\right)=0$ unless $H \supseteq{ }^{g} K$ and also ${ }^{g} K \supseteq H$, or in other words $H={ }^{g} K$.

Finally (c) is proved dually to (a). We may also deduce it from (a) since when $R$ is a field $\nabla_{H, U} \cong\left(\Delta_{H, U^{*}}\right)^{*}$ and $\operatorname{Ext}\left(\nabla_{H, U}, \nabla_{K, W}\right) \cong \operatorname{Ext}\left(\Delta_{K, W^{*}}, \Delta_{H, U^{*}}\right)$ which equals 0 unless $H<_{G} K$ by (a). 
Improvements on 5.1 seem hard to come by. For example, we might hope that $\operatorname{Ext}_{\mu_{R}(G)}^{1}\left(\Delta_{H, U}, \nabla_{K, W}\right)=0$ always, in the same manner as for a quasi-hereditary algebra [10], but this is not so. When $G=C_{2}$ and $R$ is the field of two elements we calculate directly that $\operatorname{Ext}_{\mu_{R}(G)}^{1}\left(\Delta_{1, R}, \nabla_{1, R}\right) \cong R$.

In the same spirit as 5.1 we present a similar result about homomorphisms.

(5.2) PROPOSITION. Let $H$ and $K$ be subgroups of $G$ and let $U$ and $W$ be $p$ permutation modules for $N_{G}(H) / H$ and $N_{G}(K) / K$ respectively. Then

(a) $\operatorname{Hom}_{\mu_{R}(G)}\left(\Delta_{H, U}, \Delta_{K, W}\right)=0$ unless $H \geq_{G} K$.

(b) $\operatorname{Hom}_{\mu_{R}(G)}\left(\Delta_{H, U}, \nabla_{K, W}\right)=0$ unless $H=_{G} K$.

(c) $\operatorname{Hom}_{\mu_{R}(G)}\left(\nabla_{H, U}, \nabla_{K, W}\right)=0$ unless $H \leq_{G} K$.

Proof. If $M$ is any Mackey functor for which $M(H)=0$ then $\operatorname{Hom}_{\mu_{R}(G)}\left(\Delta_{H, U}, M\right)=$ 0 since $\Delta_{H, U}$ is generated by its value at $H$, and if $f: \Delta_{H, U} \rightarrow M$ is non-zero then $f\left(\Delta_{H, U}\right)(H)$ must be non-zero also. Since $\Delta_{K, W}(H)=\nabla_{K, W}(H)=0$ unless $H \geq_{G}$ $K$ this proves (a) and establishes half of (b). Dually, if $M$ is any Mackey functor for which $M(K)=0$ then $\operatorname{Hom}_{\mu_{R}(G)}\left(M, \nabla_{K, W}\right)=0$ since $\nabla_{K, W}$ is cogenerated at $K$. Since $\Delta_{H, U}(K)=\nabla_{H, U}(K)=0$ unless $H \leq_{G} K$ this proves (c) and establishes the other half of (b).

\section{6. $\mathcal{D}$ has relative almost split sequences}

As a step towards proving that $\mathcal{D}$ has relative almost split sequences we need to know that $\mathcal{D}$ is closed under taking direct summands, and we prove this in the next results. In the first, we show that any $\Delta$-filtration is compatible, in a certain sense, with any ascending filtration. We continue to suppose that $R$ is a complete $p$-local ring.

(6.1) PROPOSITION. Let $1=H_{1}, H_{2}, \ldots, H_{n}=G$ be a list of representatives of the conjugacy classes of subgroups of $G$ with the property that whenever $H_{i}$ is conjugate to a subgroup of $H_{j}$ then $i \leq j$. Let $M$ be a Mackey functor in $\mathcal{D}$. Let $M_{i}=I_{\left\{H_{1}, \ldots, H_{i}\right\}} M$ be the terms of the corresponding ascending filtration.

(a) We have $M_{i} / M_{i-1} \cong \Delta_{H_{i}, \bar{M}\left(H_{i}\right)}$ where $\bar{M}\left(H_{i}\right)$ is a $p$-permutation module for $N_{G}\left(H_{i}\right) / H_{i}$. Thus the ascending filtration of $M$ is a $\Delta$-filtration.

(b) If $\cdots \subseteq F_{k} \subseteq F_{k+1} \subseteq \cdots \subseteq M$ is any filtration of $M$ with indecomposable $\Delta$ factors then the terms of the filtration may be changed one by one in the fashion $F_{k} \rightarrow F_{k}^{\prime}$ where

$$
\begin{aligned}
F_{k+1} / F_{k-1} & \cong F_{k+1} / F_{k} \oplus F_{k} / F_{k-1} \\
& \cong F_{k}^{\prime} / F_{k-1} \oplus F_{k+1} / F_{k}^{\prime}
\end{aligned}
$$

such that we start with the given filtration and finish with a refinement of the filtration whose terms are the $M_{i}$. 
(c) The multiplicity of each indecomposable $\Delta_{H_{i}, U_{i j}}$ in any filtration of $M$ with $\Delta$ factors is determined independently of the choice of filtration, and equals the multiplicity of $U_{i j}$ as a summand of $\bar{M}\left(H_{i}\right)$.

Proof. We first prove (b). Because $\operatorname{Ext}\left(\Delta_{H, U}, \Delta_{K, W}\right)=0$ unless $H>_{G} K$, starting with any filtration $\cdots \subseteq F_{k} \subseteq F_{k+1} \subseteq \cdots$ we may perform a sequence of changes in an inductive fashion starting with all the factors of the form $F_{k+1} / F_{k} \cong \Delta_{H_{1}, U_{1 j}}$ indexed by $H_{1}$ and moving them to the bottom of the filtration, then moving all the factors $F_{k+1} / F_{k} \cong \Delta_{H_{2}, U_{2 j}}$ immediately above them, and so on until all $\Delta_{H_{i}, U_{i j}}$ factors occur together for a given $i$, after the ones for $i-1$ and before $i+1$. If $F_{k}$ is the term that has beneath it all the $\Delta_{H_{r}, U_{r j}}$ factors with $r \leq i$ then $F_{k}$ is generated by its values at the $H_{r}$ with $r \leq i$ and so $F_{k} \subseteq M_{i}$. On the other hand, since $\Delta_{H_{s}, U_{s j}}\left(H_{r}\right)=0$ if $r<s$ we deduce that $M / F_{k}$ is zero on $H_{r}$ with $r \leq i$ and hence $M_{i} / F_{k}$ is zero on these $H_{r}$. But $M_{i}$ and also $M_{i} / F_{k}$ are generated by their values on such $H_{r}$, and so $M_{i} / F_{k}=0$ and hence $M_{i}=F_{k}$. This establishes (b).

(a) From what we just did we see that $M_{i} / M_{i-1}$ has a filtration by the $\Delta_{H_{i}, U_{i j}}$ indexed by $H_{i}$. These functors do not extend themselves, so the factor is a direct sum. If this sum is $\bigoplus_{j} \Delta_{H_{i}, U_{i j}}$ then it may also be written $\Delta_{H_{i}, \bigoplus_{j} U_{i j}}$ and the value of this Mackey functor at $H_{i}$ is $\left(M_{i} / M_{i-1}\right)\left(H_{i}\right)=\bigoplus_{j} U_{i j}$, which by 2.4 is $\bar{M}\left(H_{i}\right)$. This establishes (a).

(c) The multiplicity of $\Delta_{H_{i}, U_{i j}}$ in the original filtration by the $F_{k}$ is unchanged by the operations used in the proof of (b) and so equals the multiplicity of $\Delta_{H_{i}, U_{i j}}$ as a summand of $M_{i} / M_{i-1}$. We have already seen that this is the multiplicity of $U_{i j}$ as a summand of $\bar{M}\left(H_{i}\right)$. Since any $\Delta$-filtration can be transformed into a refinement of this ascending filtration without changing multiplicities, these multiplicities are independently determined.

The following is a characterization of the Mackey functors in $\mathcal{D}$ when $R$ is a field.

(6.2) COROLLARY. Let $R$ be a field. A Mackey functor $M$ is an object of $\mathcal{D}$ if and only if the following two conditions are satisfied:

(a) for all subgroups $H$ the Brauer quotient $\bar{M}(H)$ is a p-permutation $N_{G}(H) / H$-module, and

(b) for all subgroups $K$ of $G$

$$
\operatorname{dim} M(K)=\sum_{\substack{H \leq G \\ \text { up to conjugacy }}} \operatorname{dim} \Delta_{H, \bar{M}(H)}(K) .
$$

Proof. Let $1=H_{1}, H_{2}, \ldots, H_{n}=G$ be a list of representatives of the conjugacy classes of subgroups of $G$ with the property that whenever $H_{i}$ is conjugate to a subgroup of $H_{j}$ then $i \leq j$ and let $M_{i}=I_{\left\{H_{1}, \ldots, H_{i}\right\}} M$. Then we know in general from 3.6 that

$$
\operatorname{dim} M(K) \leq \sum_{i=1}^{n} \operatorname{dim} \Delta_{H_{i}, \bar{M}\left(H_{i}\right)}(K)
$$


and that we have equality if and only if every factor $M_{i} / M_{i-1}$ is isomorphic to $\Delta_{H_{i}, \bar{M}\left(H_{i}\right)}$. The only remaining condition for $M$ to lie in $\mathcal{D}$ is supplied by (a).

The following result is also clear from our analysis.

(6.3) COROLLARY. Let $M$ be a Mackey functor in $\mathcal{D}$ and suppose that a particular subgroup $H$ appears as $H_{i}$ in a list $1=H_{1}, H_{2}, \ldots, H_{n}=G$ and as $K_{j}$ in a list $1=K_{1}, K_{2}, \ldots, K_{n}=G$ of conjugacy classes of subgroups, where both lists satisfy the condition that the conjugates of subgroups of each term appear earlier in the list. Let $M_{i}$ and $M_{j}^{\prime}$ denote the terms of the corresponding ascending filtrations of $M$. Then $M_{i} / M_{i-1} \cong M_{j}^{\prime} / M_{j-1}^{\prime}$. In other words, the factor associated to $H$ does not depend on the position of $H$ in the list.

It is the following corollary which will in fact be used to deduce the main result of this section.

(6.4) COROLlary. Let $M=A \oplus B$ be a functor in $\mathcal{D}$. Then $A$ and $B$ also lie in $\mathcal{D}$.

Proof. We have $M_{i}=A_{i} \oplus B_{i}$ and so $M_{i} / M_{i-1}=\bigoplus \Delta_{H_{i}, U_{i j}}^{m_{i j}}=A_{i} / A_{i-1} \oplus B_{i} / B_{i-1}$. Since the $\Delta_{H_{i}, U_{i j}}$ are indecomposable, by the Krull-Schmidt theorem we have that each of $A^{i} / A^{i-1}$ and $B^{i} / B^{i-1}$ is a direct sum of functors $\Delta_{H_{i}, U_{i j}}$ and so $A$ and $B$ are objects in $\mathcal{D}$.

We come to one of the principal results of this paper. It relies on the work of [10] which deals with representations of artin algebras. Because of this we require $R$ to be a field.

(6.5) THEOREM. Let $R$ be a field. The category $\mathcal{D}$ is functorially finite in $\operatorname{Mack}_{R}(G)$, and has relative almost split sequences.

Proof. This is a consequence of Theorem 1 and Corollary 1 of [10]. That theorem has to do with a subcategory of modules with filtrations with factors in a list $\Theta(1), \ldots, \Theta(n)$ where $\operatorname{Ext}(\Theta(j), \Theta(i))=0$ for $j \geq i$. If we arrange our Mackey functors $\Delta_{H, U}$ with $U$ a $p$-permutation module in a list so that whenever $K$ is conjugate to a subgroup of $H$ then $\Delta_{H, U}$ precedes $\Delta_{K, W}$, this list will satisfy Ringel's condition by Theorem 5.1. Ringel's theorem now shows that $\mathcal{D}$ is functorially finite in $\operatorname{Mack}_{R}(G)$. Because $\mathcal{D}$ is closed under taking direct summands, Corollary 1 of [10] says that $\mathcal{D}$ has relative almost split sequences. 


\section{Projective Mackey functors lie in $\mathcal{D}$}

We now show that projective Mackey functors are objects in $\mathcal{D}$. The proof uses the formal machinery we have developed, and for this reason appears late in this exposition. We have also been able to find a more direct argument which could have been presented earlier, but it is longer and requires quite elaborate combinatorial manipulation. It seems less satisfactory conceptually than the one we are about to give.

(7.1) THEOREM. Let $M$ be a finitely-generated projective Mackey functor over a complete $p$-local ring. Then $M$ is an object in $\mathcal{D}$.

Proof. We start with the same reduction that was made at the start of the proof of 5.1, namely we may assume that our projective Mackey functor $M$ lies in $\operatorname{Mack}_{R}(G, 1)$. For suppose that $M$ happens to lie in $\operatorname{Mack}_{R}(G, J)$ where $J$ is a $p$-perfect subgroup of $G$. The reverse functor in the equivalence

$$
\left(\operatorname{Inf}_{N(J) / J}^{N(J)}-\right) \uparrow_{N(J)}^{G}: \operatorname{Mack}_{R}(N(J) / J, 1) \rightarrow \operatorname{Mack}_{R}(G, J)
$$

is restriction followed by deflation. If we regard $M$ as a functor in $\operatorname{Mack}_{R}\left(N_{G}(J) / J, 1\right)$ by this means and produce a $\Delta$-filtration of it in this category, then on applying $\left(\operatorname{Inf}_{N(J) / J}^{N(J)}-\right) \uparrow_{N(J)}^{G}$ we will obtain a $\Delta$-filtration of $M$ in $\operatorname{Mack}_{R}(G, J)$, by Lemmas 4.7 and 4.1. In view of this we will now assume that $M \in \operatorname{Mack}_{R}(G, 1)$.

We next claim that it suffices to assume $M=B^{Q} \uparrow_{Q}^{G}$ for some $p$-subgroup $Q$, where $B^{Q}$ is the Burnside ring Mackey functor for the subgroup $Q$. This is because every projective functor in $\operatorname{Mack}_{R}(G, 1)$ is a direct summand of a direct sum of functors of the form $B^{Q} \uparrow_{Q}^{G}$ with $Q$ a $p$-group [14, 8.7], and direct summands of objects in $\mathcal{D}$ again lie in $\mathcal{D}$ by 6.4.

In order to show that $B^{Q} \uparrow_{Q}^{G}$ has a $\Delta$-filtration it suffices to show that $B^{Q}$ has a $\Delta$ filtration, as a Mackey functor for the $p$-group $Q$. This is because the property of having a $\Delta$-filtration is preserved under induction, by 4.8 .

Let $1=H_{1}, H_{2}, \ldots, H_{n}=Q$ be representatives of the conjugacy classes of subgroups of $Q$, listed so that whenever $H_{i}$ is conjugate to a subgroup of $H_{j}$ then $i \leq j$. We will show that the corresponding ascending filtration of $B^{Q}$ is a $\Delta$-filtration by showing that each of the epimorphisms

$$
\Delta_{H_{i}, \bar{B}_{\left(H_{i}\right)}} \rightarrow\left(B^{Q}\right)_{i} /\left(B^{Q}\right)_{i-1}
$$

of Corollary 3.5 is an isomorphism and also that $\overline{B^{Q}}\left(H_{i}\right)$ is a $p$-permutation $R\left[N_{Q}\left(H_{i}\right) / H_{i}\right]$ module for all subgroups $H_{i}$ of $Q$. In fact $\overline{B^{Q}}\left(H_{i}\right)=R$ since all $H_{i}$-sets except the one-point set are induced from proper subgroups; and $R$ is indeed a $p$-permutation module. Thus it 
remains to show that the epimorphisms are isomorphisms, and we claim that it is sufficient to show that

$$
\sum_{i=1}^{n} \operatorname{rank}_{R} \Delta_{H_{i}, \bar{B}^{Q}\left(H_{i}\right)}(K)=\operatorname{rank}_{R} B^{Q}(K) \text { for every subgroup } K \text { of } Q .
$$

To see why this is so, we note from Proposition 3.1 that in this particular situation

$$
\Delta_{H_{i}, \bar{B}^{Q}\left(H_{i}\right)}(K)=\bigoplus_{g \in\left[K \backslash T\left(H_{i}, K\right) / N_{Q}\left(H_{i}\right)\right]} R
$$

for each subgroup $K$, and this is a free $R$-module. Also $\left(B^{Q}\right)_{i}(K)$ is the span in $B(K)$ of the $K$-sets $K / H_{j}$ where $j \leq i$ and $H_{j} \subseteq K$, so that $\left(B^{Q}\right)_{i} /\left(B^{Q}\right)_{i-1}(K)$ is also a free $R$-module. Thus to show that the epimorphisms are isomorphisms it suffices to show that

$$
\operatorname{rank}_{R} \Delta_{H_{i}, \overline{B^{Q}}\left(H_{i}\right)}(K)=\operatorname{rank}_{R}\left(\left(B^{Q}\right)_{i} /\left(B^{Q}\right)_{i-1}(K)\right)
$$

for all $H_{i}$ and for all subgroups $K$ of $Q$. Since the rank on the left is always at least the rank on the right, it suffices to show that

$$
\sum_{i=1}^{n} \operatorname{rank} \Delta_{H_{i}, \bar{B}^{Q}\left(H_{i}\right)}(K)=\sum_{i=1}^{n} \operatorname{rank}_{R}\left(\left(B^{Q}\right)_{i} /\left(B^{Q}\right)_{i-1}(K)\right) \quad \text { for every subgroup } K \text { of } Q
$$

and the quantity on the right is $\operatorname{rank} B^{Q}(K)$. This establishes the most recent claim.

Now to verify that the claimed equality is indeed so we use again the formula from Proposition 3.1 to obtain

$$
\operatorname{rank}_{R} \Delta_{H_{i}, R}(K)=\left|K \backslash T\left(H_{i}, K\right) / N_{Q}\left(H_{i}\right)\right|
$$

This quantity is well known to be the number of $K$-conjugacy classes of subgroups of $K$ which are $Q$-conjugate to $H_{i}$. The sum of these numbers over all subgroups $H_{i}$ (taken up to $Q$-conjugacy) equals the number of $K$-conjugacy classes of subgroups of $K$ since these are partitioned by the relation of being conjugate in $Q$. This in turn is equal to rank $B^{Q}(K)$, which completes the proof.

We point out that the factors in a $\Delta$-filtration of a projective Mackey functor $M$ may be determined by combining results from [14] and [3] with Proposition 6.1. For by 6.1(a) $M$ has a filtration with factors $\Delta_{H, \bar{M}(H)}$, and assuming that $M \in \operatorname{Mack}_{R}(G, 1)$ the modules $\bar{M}(H)$ are determined in $[3,5.10]$ as Brauer quotients

$$
M(1)^{H} / \sum_{J<H} \operatorname{tr}_{J}^{H} M(1)^{J}
$$

Finally if $M$ is indecomposable and has simple quotient $S_{K, W}$, then $M(1)$ is the Green correspondent of the projective cover (as a $R\left[N_{G}(K) / K\right]$-module) of $V$, by $[14,12.7$ (ii)]. 


\section{Ext-projective and Ext-injective objects in $\mathcal{D}$}

In this section and the next we go further with the methods used by Ringel [10] and extend them to a situation where weaker hypotheses are in force than the ones he considered. Our general set-up will be the following situation:

(8.1) HYPOTHESIS. $\mathcal{D}$ is the full subcategory of the finite length modules for an artin algebra $\mu$ whose objects are the modules with a filtration whose factors lie in a set $\{\Theta(\lambda) \mid \lambda \in \Lambda\}$, where $\Lambda$ is a finite pre-ordered set.

A pre-ordered set (also called a quasi-poset) is a set with a reflexive, transitive binary relation $\leq$. We write $\rho<\lambda$ if $\rho \leq \lambda$ and $\lambda \not \leq \rho$. In our application to Mackey functors we will take $\Lambda$ to be the set of pairs $(H, U)$ where $H$ is a subgroup of $G$ taken up to conjugacy and $U$ is an indecomposable $p$-permutation $R\left[N_{G}(H) / H\right]$-module taken up to isomorphism. The pre-order on this set is specified by $(H, U) \leq(K, V)$ if and only if $H$ is conjugate to a subgroup of $K$. Then $(H, U)<(K, V)$ will mean that $H$ is conjugate to a proper subgroup of $K$.

In Ringel's work [10] the modules $\Theta(\lambda)$ were linearly ordered, rather than being related by a pre-order. We may still apply his results here by putting the elements of $\Lambda$ in order $\Lambda=\left\{\lambda_{1}, \ldots, \lambda_{n}\right\}$ in such a way that $\lambda_{i}>\lambda_{j}$ implies $i<j$ (we deliberately choose to place the $\lambda$ 's in non-ascending order so as to be consistent with Ringel). For example, we will work with the condition that $\operatorname{Ext}(\Theta(\lambda), \Theta(\rho)) \neq 0$ implies $\lambda>\rho$. If we place the elements of $\Lambda$ in a total order in this way, this condition implies $\operatorname{Ext}\left(\Theta\left(\lambda_{i}\right), \Theta\left(\lambda_{j}\right)\right)=0$ unless $i<j$, which is the condition which Ringel required in [10].

We begin with some generalities about Ext-projectivity and Ext-injectivity. We say that an object $M$ of $\mathcal{D}$ is Ext-injective if $\operatorname{Ext}(A, M)=0$ for all objects $A$ in $\mathcal{D}$, and it is Ext-projective if $\operatorname{Ext}(M, A)=0$ for all objects $A$ in $\mathcal{D}$. Although we only really work explicitly with Ext-injective objects in this section, all of our results have dual versions which relate to Ext-projective objects, since there is nothing in our hypotheses to favour injectivity rather than projectivity.

We start by showing that Ext-injective objects have familiar lifting and splitting properties.

(8.2) LEMMA. In the situation of Hypothesis 8.1, let I be an Ext-injective object in $\mathcal{D}$.

(a) Given mappings

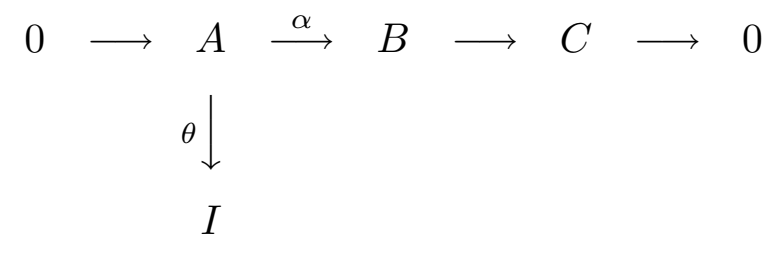


in which the row is a short exact sequence and all objects lie in $\mathcal{D}$, there exists a mapping $\beta: B \rightarrow I$ so that $\theta=\beta \alpha$.

(b) Every short exact sequence $0 \rightarrow I \rightarrow B \rightarrow C \rightarrow 0$ in which all objects belong to $\mathcal{D}$ is split.

Proof. (a) Consider the long exact sequence

$$
0 \rightarrow \operatorname{Hom}(C, I) \rightarrow \operatorname{Hom}(B, I) \rightarrow \operatorname{Hom}(A, I) \rightarrow \operatorname{Ext}(C, I)=0
$$

This shows that the mapping $\theta$ is the image of a mapping $\beta: B \rightarrow I$.

(b) We apply (a) with $A=I$, taking $\theta$ to be the identity map on $I$.

We will say that a morphism $A \stackrel{\alpha}{\longrightarrow} I$ of objects in $\mathcal{D}$ is an Ext-injective hull of $A$ if the following four conditions are satisfied:

1. $\alpha$ is a monomorphism.

2. $I$ is Ext-injective.

3. Coker $\alpha$ is an object in $\mathcal{D}$.

4. Whenever $\beta: I \rightarrow J$ is a morphism where $J$ is an object of $\mathcal{D}, \beta \alpha$ is a monomorphism and Coker $\beta \alpha$ is an object of $\mathcal{D}$ then $\beta$ is a monomorphism.

We define an Ext-projective cover by the similar dual property.

(8.3) PROPOSITION. In the situation of Hypothesis 8.1, if an object of $\mathcal{D}$ has an Ext-injective hull it is unique up to isomorphism. Similarly, if an object of $\mathcal{D}$ has an Ext-projective cover it is unique up to isomorphism.

Proof. Let $\alpha: A \rightarrow I$ and $\alpha^{\prime}: A \rightarrow I^{\prime}$ be two Ext-injective hulls of $A$. We show that they are isomorphic. By Ext-injectivity we can find $\beta: I \rightarrow I^{\prime}$ with $\alpha^{\prime}=\beta \alpha$ and $\gamma: I^{\prime} \rightarrow I$ with $\alpha=\gamma \alpha^{\prime}$. The second equation shows that $\gamma$ is a monomorphism by condition 4 . Putting the two equations together gives $\alpha=\gamma \beta \alpha$ which shows that $\gamma \beta: I \rightarrow I$ is a monomorphism, again by condition 4 . Since $I$ has finite length, it follows that $\gamma \beta$ is an isomorphism, and hence $\gamma$ is an epimorphism. This shows that $\gamma$ is an isomorphism, and so the two Ext-injective hulls are isomorphic.

The uniqueness of Ext-projective covers is proved dually.

The argument we have just seen will also prove the following statement, whose dual formulation for Ext-projectivity we leave to the reader.

(8.4) PROPOSITION. Let $\alpha: A \rightarrow I$ be an Ext-injective hull of $A$ in $\mathcal{D}$, and let $\alpha^{\prime}: A \rightarrow I^{\prime}$ be any monomorphism to an Ext-injective object $I^{\prime}$ such that Coker $\alpha^{\prime}$ is an object in $\mathcal{D}$. Then there is a morphism $\beta: I \rightarrow I^{\prime}$ so that $\alpha^{\prime}=\beta \alpha$, and furthermore any such $\beta$ is a split monomorphism.

Proof. As in the last result we construct $\beta: I \rightarrow I^{\prime}$ with $\alpha^{\prime}=\beta \alpha$ and $\gamma: I^{\prime} \rightarrow I$ with $\gamma \alpha^{\prime}=\alpha$. Thus $\alpha=\gamma \beta \alpha$ and by condition 4 we deduce that $\gamma \beta: I \rightarrow I$ is a monomorphism. Hence $\gamma \beta$ is an isomorphism since $I$ has finite length, and so $\beta$ is a split monomorphism. 
We now come to the existence of Ext-injective hulls and Ext-projective covers, and for this we impose further hypotheses on top of 8.1 .

(8.5) PROPOSITION. In the situation of Hypothesis 8.1, suppose further that direct summands of modules in $\mathcal{D}$ again lie in $\mathcal{D}$ and that for all $\lambda, \rho \in \Lambda$ we have

$$
\operatorname{Ext}(\Theta(\lambda), \Theta(\rho))=0
$$

unless $\lambda>\rho$. Then each object $A$ in $\mathcal{D}$ has an Ext-injective hull and an Ext-projective cover in $\mathcal{D}$.

Proof. Let us start by writing $\Lambda=\left\{\lambda_{1}, \ldots, \lambda_{n}\right\}$ in such a way that $\lambda_{i}>\lambda_{j}$ implies $i<j$. Then $\operatorname{Ext}\left(\Theta\left(\lambda_{i}\right), \Theta\left(\lambda_{j}\right)\right)=0$ unless $i<j$ and we may apply the results of [10]. From Lemma $4^{\prime}$ of [10] we see that it is possible to find a mapping $\alpha: A \rightarrow I$ to an object of $\mathcal{D}$ satisfying conditions 1,2 and 3 of the definition of an Ext-injective hull. Among such mappings, choose one with $I$ of minimal length. We show that condition 4 is satisfied. Suppose $\beta: I \rightarrow J$ is a morphism as in condition 4 . Then by the Ext-injective property there exists $\gamma: J \rightarrow I$ with $\alpha=\gamma \beta \alpha$ and hence $\alpha=(\gamma \beta)^{n} \alpha$ for every $n \geq 1$. By Fitting's lemma there exists $n$ so that $I=(\gamma \beta)^{n} I \oplus \operatorname{Ker}(\gamma \beta)^{n}$ and now $\alpha$ factorizes as $A \stackrel{\alpha_{1}}{\longrightarrow}(\gamma \beta)^{n} I \hookrightarrow I$. We show that $A \stackrel{\alpha_{1}}{\longrightarrow}(\gamma \beta)^{n} I$ satisfies conditions 1,2 and 3 of the definition of an Ext-injective hull. First, $(\gamma \beta)^{n} I$ is an object of $\mathcal{D}$, since it is a direct summand of $I$, and for the same reason it is Ext-injective. Also, $\alpha_{1}$ is a monomorphism and Coker $\alpha_{1}$ appears in the exact sequence $0 \rightarrow \operatorname{Ker}(\gamma \beta)^{n} \rightarrow$ Coker $\alpha_{1} \rightarrow$ Coker $\alpha \rightarrow 0$, which shows that Coker $\alpha_{1}$ is an object in $\mathcal{D}$ since $\operatorname{Ker}(\gamma \beta)^{n}$ is a summand of $I$ and so lies in $\mathcal{D}$. It follows by minimality of $I$ that $I=(\gamma \beta)^{n} I$ and hence $\beta$ is a monomorphism. This shows that condition 4 is satisfied.

It is clear that Ext-projective covers may be produced by a dual construction.

We will denote by $T(\lambda)$ the Ext-injective hull in $\mathcal{D}$ of $\Theta(\lambda)$, and by $\Pi(\lambda)$ the Extprojective cover in $\mathcal{D}$ of $\Theta(\lambda)$.

(8.6) THEOREM. In the situation of Hypothesis 8.1, suppose further that direct summands of modules in $\mathcal{D}$ again lie in $\mathcal{D}$ and that for all $\lambda, \rho \in \Lambda$ we have

$$
\operatorname{Ext}(\Theta(\lambda), \Theta(\rho))=0
$$

unless $\lambda>\rho$. Then the objects $T(\lambda)$ are precisely the indecomposable Ext-injective objects in $\mathcal{D}$. Each $T(\lambda)$ has the property that in any filtration with $\Theta$ factors, $\Theta(\lambda)$ always occurs as the bottom factor, and the other $\Theta(\rho)$ factors all have $\rho<\lambda$. The objects $\Pi(\lambda)$ are precisely the indecomposable Ext-projective objects in $\mathcal{D}$. Each $\Pi(\lambda)$ has the property 
that in any filtration with $\Theta$ factors, $\Theta(\lambda)$ always occurs as the top factor, and the other $\Theta(\rho)$ factors all have $\rho>\lambda$.

Proof. We first show that the $T(\lambda)$ are the only indecomposable Ext-injectives in $\mathcal{D}$. Suppose that $J$ is an indecomposable Ext-injective in $\mathcal{D}$. Then there is a short exact sequence $0 \rightarrow \Theta(\lambda) \rightarrow J \rightarrow C \rightarrow 0$ with $C$ in $\mathcal{D}$, for some $\lambda$. Let $\Theta(\lambda) \rightarrow T(\lambda)$ be the Ext-injective hull of $\Theta(\lambda)$. By 8.4 there exists a morphism $\beta: T(\lambda) \rightarrow J$ which is a split monomorphism. Since $J$ is indecomposable we deduce that $J \cong T(\lambda)$. This shows that the list of indecomposable Ext-injective objects in $\mathcal{D}$ is complete. The $T(\lambda)$ have the claimed properties by the construction in Lemma $4^{\prime}$ of [10], and by the vanishing of Ext groups between the $\Theta(\rho)$.

The proof of the statement about Ext-projectives is dual.

In the specific case of the category $\mathcal{D}$ of Mackey functors whose objects are the Mackey functors with $\Delta$-filtrations, we denote by $T_{H, U}$ the Ext-injective hull of $\Delta_{H, U}$ and $\Pi_{H, U}$ the Ext-projective cover of $\Delta_{H, U}$. By 8.6 we get a complete list of indecomposable Extinjectives and Ext-projectives in $\mathcal{D}$ as $(H, U)$ ranges through all pairs with $H$ taken up to conjugacy and the indecomposable $p$-permutation $R\left[N_{G}(H) / H\right]$-module $U$ taken up to isomorphism.

\section{A standardly stratified algebra}

The similarity between the properties of Mackey functors in $\mathcal{D}$ and the modules with a good filtration of Ringel [10] allows us to imitate one of his main constructions, that of the 'Ringel dual'. In the case of a quasi-hereditary algebra the modules with a $\Delta$ filtration which also have a $\nabla$-filtration coincide with the Ext-injective modules with a $\Delta$ filtration. The Ringel dual is the endomorphism ring of the direct sum of these canonical indecomposable modules. In the generality of the last section having to do with modules for a more general artin algebra $\mu$, we replace the condition that modules have both a $\Delta$ and a $\nabla$-filtration by the condition that they are Ext-injective, which comes to the same thing for a quasi-hereditary algebra. Using these modules which we classified in Theorem 8.6, we show with one further hypothesis that the endomorphism ring of their direct sum is standardly stratified in the sense of [4].

(9.1) THEOREM. In the situation of Hypothesis 8.1, suppose that $\mathcal{D}$ is closed under taking direct summands, that $\operatorname{Ext}(\Theta(\lambda), \Theta(\rho))=0$ unless $\lambda>\rho$, and also that $\operatorname{Hom}(\Theta(\lambda), \Theta(\rho))=0$ unless $\lambda>\rho$. Let $T=\bigoplus_{\lambda \in \Lambda} T(\lambda)$ be the direct sum of all the isomorphism types of indecomposable Ext-injective objects in $\mathcal{D}$. Then the algebra $E=\operatorname{End}_{\mu}(T)$ is standardly stratified in the sense of [4] with pre-ordered set $\Lambda$.

Proof. We verify the conditions of Definition 2.2.1 of [4]. The indecomposable projective modules for $E$ are $Q(\lambda)=\operatorname{Hom}_{\mu}(T(\lambda), T)$ and we define $\Delta(\lambda)=\operatorname{Hom}_{\mu}(\Theta(\lambda), T)$. 
The homomorphism $Q(\lambda) \rightarrow \Delta(\lambda)$ which comes by functoriality from the inclusion $\Theta(\lambda) \rightarrow$ $T(\lambda)$ is epi since $T$ is Ext-injective. Let us denote the simple $E$-module which is the unique simple quotient of $Q(\lambda)$ by $L(\lambda)$.

We will use the following result, which is similar to Yoneda's lemma.

(9.2) LEMMA. $\operatorname{Hom}_{E}(Q(\rho), \Delta(\lambda)) \cong \operatorname{Hom}_{\mu}(\Theta(\lambda), T(\rho))$.

Proof. We show that the homomorphism

$$
\begin{aligned}
\operatorname{Hom}_{\mu}(\Theta(\lambda), T(\rho)) & \rightarrow \operatorname{Hom}_{E}(\operatorname{Hom}(T(\rho), T), \operatorname{Hom}(\Theta(\lambda), T)) \\
\alpha & \mapsto(\theta \mapsto \theta \circ \alpha)
\end{aligned}
$$

is an isomorphism. It is a monomorphism because we may take $\theta$ to be the inclusion $\iota$ : $T(\rho) \hookrightarrow T$ and then $\theta \circ \alpha=0$ implies $\alpha=0$. It is an epimorphism: let $\phi: \operatorname{Hom}(T(\rho), T) \rightarrow$ $\operatorname{Hom}(\Theta(\lambda), T)$ and consider $\phi(\iota): \Theta(\lambda) \rightarrow T$. We show that $\phi(\iota)$ factors through $\iota$. Let $\pi: T \rightarrow T$ be the identity on $T(\rho)$ and 0 on the other summands. Then $\pi \iota=\iota$. Also $\phi(\pi \iota)=\pi \phi(\iota)$ since $\phi$ is an $E$-map. Thus $\phi(\iota)=\pi \phi(\iota)$ so $\phi(\iota)$ has image contained in $\iota(T(\rho))$. Write $\phi(\iota)=\iota \bar{\phi}$ where $\bar{\phi}: \Theta(\lambda) \rightarrow T(\rho)$. We show that $\phi$ is the image of $\bar{\phi}$. To see this, let $\beta: T(\rho) \rightarrow T$ and let $\tilde{\beta}: T \rightarrow T$ be $\beta$ on $T(\rho)$, and 0 on the other summands. Then $\phi(\beta)=\phi(\tilde{\beta} \iota)=\tilde{\beta} \phi(\iota)=\tilde{\beta} \iota \bar{\phi}=\beta \bar{\phi}$ shows that $\phi$ is the image of $\bar{\phi}$. This completes the proof.

We verify condition (1) of Definition 2.2.1 of [4], which says the following: if the composition factor multiplicity $[\Delta(\lambda): L(\rho)]$ is non-zero then $\rho \leq \lambda$. This composition factor multiplicity is non-zero precisely if $\operatorname{Hom}_{\mu}(Q(\rho), \Delta(\lambda))$ is non-zero, which by Lemma 9.2 is non-zero precisely if $\operatorname{Hom}_{\mu}(\Theta(\lambda), T(\rho)) \neq 0$. All $\Theta$-factors of $T(\rho)$ are $\Theta(\sigma)$ with $\sigma \geq \rho$, and since $\operatorname{Hom}_{\mu}(\Theta(\lambda), \Theta(\sigma))=0$ unless $\lambda \geq \sigma$ we have $\operatorname{Hom}_{\mu}(\Theta(\lambda), T(\rho))=0$ unless $\rho \leq \lambda$. This shows that condition (1) is satisfied.

We now verify condition (2) of Definition 2.2.1 of [4], which says the following in our situation: for each $\lambda \in \Lambda$ there is a short exact sequence $0 \rightarrow R(\lambda) \rightarrow Q(\lambda) \rightarrow \Delta(\lambda) \rightarrow 0$ where $R(\lambda)$ has a filtration with factors $\Theta(\rho)$ with $\rho>\lambda$. By construction of the $T(\lambda)$, each one has a filtration $0=F_{0} T(\lambda) \subseteq F_{1} T(\lambda) \subseteq \cdots \subseteq F_{n} T(\lambda)=T(\lambda)$ with $F_{1} T(\lambda) \cong \Theta(\lambda)$ and $F_{i} T(\lambda) / F_{i-1} T(\lambda) \cong \Theta_{\rho_{i}}$ with $\rho_{i}>\lambda$. Now we get a filtration $0=F_{n} Q(\lambda) \subseteq F_{n-1} Q(\lambda) \subseteq$ $\cdots \subseteq F_{0} Q(\lambda)=Q(\lambda)$ where $F_{i} Q(\lambda)=\operatorname{Ker}\left(\operatorname{Hom}(T(\lambda), T) \rightarrow \operatorname{Hom}\left(F_{i} T(\lambda), T\right)\right)$. Consider the following commutative diagram, in which the rows are exact at the right since $T$ is 
Ext-injective.

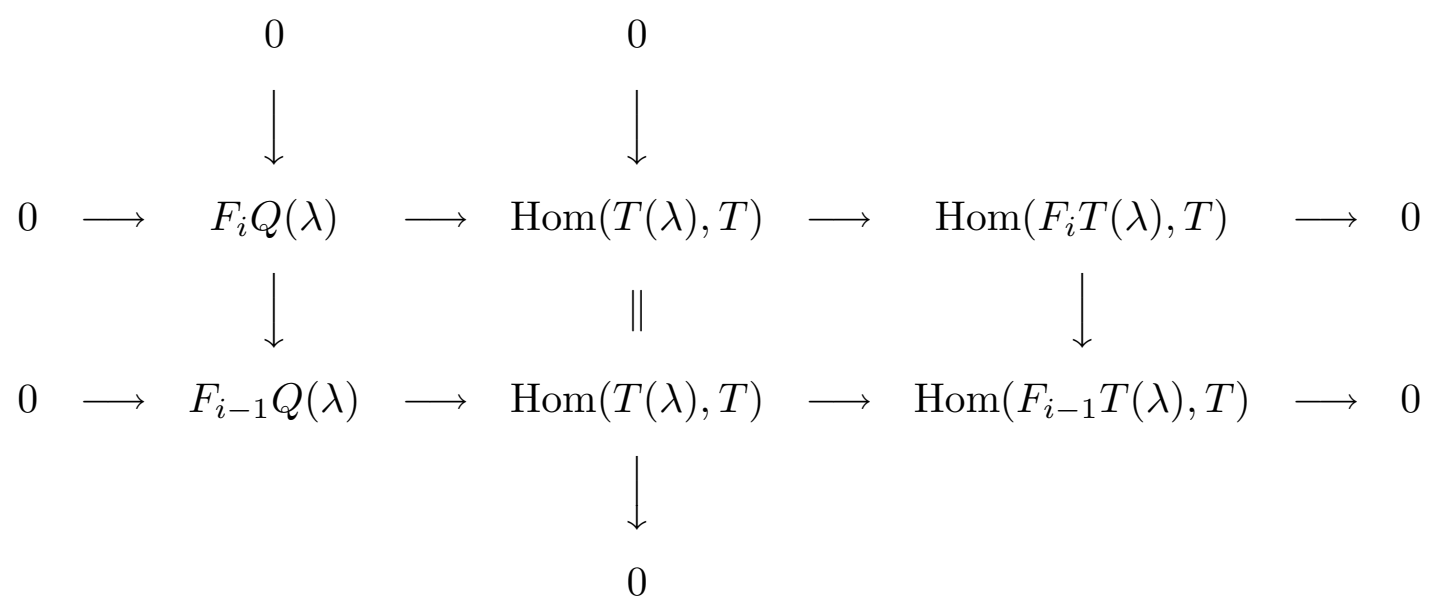

By the snake lemma we have

$$
F_{i-1} Q(\lambda) / F_{i} Q(\lambda) \cong \operatorname{Ker}\left(\operatorname{Hom}\left(F_{i} T(\lambda), T\right) \rightarrow \operatorname{Hom}\left(F_{i-1} T(\lambda), T\right)\right) .
$$

Now $0 \rightarrow \operatorname{Hom}\left(F_{i} T(\lambda) / F_{i-1} T(\lambda), T\right) \rightarrow \operatorname{Hom}\left(F_{i} T(\lambda), T\right) \rightarrow \operatorname{Hom}\left(F_{i-1} T(\lambda), T\right)$ is exact, so

$$
F_{i-1} Q(\lambda) / F_{i} Q(\lambda) \cong \operatorname{Hom}\left(F_{i} T(\lambda) / F_{i-1} T(\lambda), T\right) \cong \Delta\left(\rho_{i}\right) .
$$

This gives a filtration of $Q(\lambda)$ by standard objects with $\Delta(\lambda)$ at the top and other factors $\Delta\left(\rho_{i}\right)$ with $\rho_{i}>\lambda$, thus completing the verification of condition (2).

In the case of our application of this theory to Mackey functors, the standardly stratified algebra $E$ stands in relation to the group algebra $R G$ in the same way as the Schur algebra stands in relation to the corresponding symmetric group algebra, as shown in the next result.

(9.3) PROPOSITION. The algebra $\operatorname{End}_{\mu_{R}(G)}\left(\bigoplus_{U \text { projective }} T_{1, U}\right)$, where the sum is taken over indecomposable $R G$-projective modules $U$, has the form eEe for a certain idempotent $e \in E$. This subalgebra is Morita equivalent to $R G$.

Proof. We have $\Delta_{1, U}=F Q_{U}$ from the definition, and if $U$ is projective this is both projective and injective as a Mackey functor by $[14,13.1]$. Thus $\Delta_{1, U}$ is Ext-injective and so $\Delta_{1, U}=T_{1, U}$. From $[13,6.1]$ we know that $\operatorname{Hom}_{\mu_{R}(G)}\left(\Delta_{1, U_{1}}, \Delta_{1, U_{2}}\right) \cong \operatorname{Hom}_{R G}\left(U_{1}, U_{2}\right)$ and so

$$
\operatorname{End}_{\mu_{R}(G)}\left(\bigoplus_{U \text { projective }} T_{1, U}\right) \cong \operatorname{End}_{R G}\left(\bigoplus_{U \text { projective }} U\right),
$$

which is Morita equivalent to $R G$. Letting $e: T \rightarrow T$ be the idempotent which is the identity on the summands $T_{1, U}$ with $U$ projective and zero on the other summands we have $e E e \cong \operatorname{End}_{\mu_{R}(G)}\left(\bigoplus_{U \text { projective }} T_{1, U}\right)$. 


\section{Two examples}

We describe the categories $\mathcal{D}$ when $G$ is the cyclic group of order 2 and the cyclic group of order 4 , and when $R$ is the field with 2 elements. Detailed arguments justifying our description will be given after we have presented all the tables. According to [13] there are two simple functors $S_{1, R}$ and $S_{C_{2}, R}$ for $C_{2}$ and three simple functors $S_{1, R}, S_{C_{2}, R}$ and $S_{C_{4}, R}$ for $C_{4}$ given explicitly by

$$
S_{H, R}(J)= \begin{cases}R & \text { if } H=J \\ 0 & \text { otherwise. }\end{cases}
$$

To shorten the notation we will simply write 1 for $S_{1, R}, 2$ for $S_{C_{2}, R}$ and 4 for $S_{C_{4}, R}$. The projective covers of these functors may be described diagrammatically as follows.

\begin{tabular}{l|ll}
$\begin{array}{l}G=C_{2} \\
(H, U)\end{array}$ & $1, R$ & $C_{2}, R$ \\
\hline \hline $\begin{array}{l}\text { Simple } \\
\text { functor }\end{array}$ & 1 & 2 \\
\hline $\begin{array}{l}\text { Projective } \\
\text { cover }\end{array}$ & 1 & 2 \\
$P_{H, U}$ & $\frac{1}{2}$ & $\frac{1}{2}$
\end{tabular}

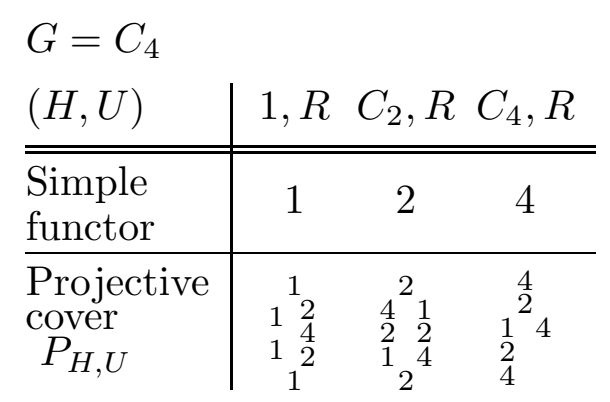

In the diagrams for these functors we indicate a non-split extension by positioning composition factors underneath each other, but in the case of $C_{4}$ not all non-split extensions are indicated in this way. The quotient of the radical by the socle of the functor $P_{C_{2}, R}$ for $C_{4}$ has the structure ${ }_{2}^{4}{ }_{1}^{4}$. In the middle of $P_{1, R}$ for $C_{4}$ there are non-split extension ${ }_{2}^{1}$ and ${ }_{1}^{2}$ in such a way that the functor $\Delta_{1, R}$ (to be described in the next table) is a homomorphic image of $P_{1, R}$, and the dual $\nabla_{1,{ }_{R}^{R}}=\Delta_{1, R}^{*}$ is a subfunctor.

We now present a description of the Mackey functors $\Delta_{H, U}$ where $U$ is a $p$-permutation module for $N_{G}(H) / H$, their Ext-injective hulls $T_{H, U}$ and Ext-projective covers $\Pi_{H, U}$ and some modules for the algebra $E=\operatorname{End}_{\mu_{R}(G)}\left(\bigoplus T_{H, U}\right)$. We will use the notation $R C_{4}$ for the induced module $R \uparrow_{1}^{C_{4}}$ and ${ }_{R}^{R}$ for the induced module $R \uparrow_{C_{2}}^{C_{4}}$, both as a representation 
of $C_{4}$ and also of $C_{4} / C_{2}$. We have the following tables of functor values:

\begin{tabular}{|c|c|c|c|}
\hline$(H, U)$ & $1, R$ & $1, \stackrel{I}{I}$ & $\mathcal{C}_{2}, R$ \\
\hline $\begin{array}{l}\text { Mackey } \\
\text { functors } \\
\Delta_{H, U} \\
\end{array}$ & $\begin{array}{l}1 \\
2\end{array}$ & $\begin{array}{l}1 \\
2 \\
1\end{array}$ & 2 \\
\hline $\begin{array}{l}\text { Ext- } \\
\text { injectives } \\
T_{H, U} \\
\end{array}$ & $\begin{array}{l}2 \\
1 \\
2\end{array}$ & $\begin{array}{l}1 \\
2 \\
1\end{array}$ & 2 \\
\hline $\begin{array}{l}\text { Ext- } \\
\text { projectives } \\
\Pi_{H, U}\end{array}$ & $\begin{array}{l}1 \\
2\end{array}$ & $\begin{array}{l}1 \\
2 \\
1\end{array}$ & $\begin{array}{l}2 \\
1 \\
2\end{array}$ \\
\hline $\begin{array}{l}\text { Simple } \\
E \text {-modules }\end{array}$ & $\alpha$ & $\beta$ & $\gamma$ \\
\hline $\begin{array}{l}\text { standard } \\
\text { modules } \\
\Delta \text { for } E \\
\end{array}$ & $\begin{array}{l}\alpha \\
\beta\end{array}$ & $\beta$ & $\begin{array}{l}\gamma \\
\alpha\end{array}$ \\
\hline $\begin{array}{l}\text { Projectives } \\
\text { for } E\end{array}$ & ${ }_{\alpha}^{\alpha} \gamma$ & $\begin{array}{l}\beta \\
\alpha \\
\beta\end{array}$ & $\begin{array}{l}\gamma \\
\alpha\end{array}$ \\
\hline
\end{tabular}

Table of Mackey functors for $C_{2}$ and modules for the stratified algebra $E$

We may check from the table for $C_{2}$ that $E$ is not only standardly stratified but in fact quasi-hereditary; however this behaviour does not seem to be typical.

\begin{tabular}{|c|c|c|c|c|c|c|}
\hline$(H, U)$ & $1, R$ & $1, \begin{array}{l}R \\
R\end{array}$ & $1, R C_{4}$ & $C_{2}, R$ & $C_{2}, \begin{array}{l}R \\
R\end{array}$ & $C_{4}, R$ \\
\hline $\begin{array}{l}\text { Mackey } \\
\text { functors } \\
\Delta_{H, U}\end{array}$ & $\begin{array}{l}1 \\
2 \\
4\end{array}$ & $\begin{array}{c}1 \\
12 \\
2\end{array}$ & $\begin{array}{l}1 \\
12 \\
14 \\
1\end{array}$ & $\begin{array}{l}2 \\
4\end{array}$ & $\begin{array}{l}2 \\
4 \\
2\end{array}$ & 4 \\
\hline $\begin{array}{l}\text { Ext- } \\
\text { injectives } \\
T_{H, U}\end{array}$ & $\begin{array}{l}\quad{ }^{4} \\
41^{2} \\
4\end{array}$ & $\begin{array}{ll} & 2 \\
4 & 1 \\
2 & 2 \\
1 & 4 \\
2 & \\
\end{array}$ & $\begin{array}{l}1 \\
12 \\
142 \\
1 \\
\end{array}$ & $\begin{array}{l}4 \\
2 \\
4\end{array}$ & $\begin{array}{l}2 \\
4 \\
2\end{array}$ & 4 \\
\hline $\begin{array}{l}\text { Ext- } \\
\text { projectives } \\
\Pi_{H, U}\end{array}$ & $\begin{array}{l}1 \\
2 \\
4\end{array}$ & $\begin{array}{c}1 \\
12 \\
2 \\
2\end{array}$ & $\begin{array}{c}1 \\
12 \\
14 \\
1 \\
1\end{array}$ & $\frac{1}{2}_{4}^{2} 4$ & $\begin{array}{l}4^{2} \\
21 \\
1 \\
1 \\
2\end{array}$ & $\begin{array}{l}{ }_{2}^{4} \\
1_{2}^{2} \\
4\end{array}$ \\
\hline $\begin{array}{l}\text { Simple } \\
E \text { - modules }\end{array}$ & $\alpha$ & $\beta$ & $\gamma$ & $\delta$ & $\epsilon$ & $\zeta$ \\
\hline $\begin{array}{l}\text { standard } \\
\text { modules } \\
\Delta \text { for } E\end{array}$ & $\begin{array}{l}\alpha \\
\beta \\
\gamma\end{array}$ & $\begin{array}{c}\beta \\
\gamma_{\beta}^{\alpha} \\
\gamma\end{array}$ & $\begin{array}{l}\gamma \\
\gamma \beta \\
\gamma \underset{\beta}{\beta} \\
\gamma\end{array}$ & $\underset{\beta}{\delta} \epsilon$ & $\underset{\beta}{\stackrel{\epsilon}{\beta} \delta}$ & $\underset{\alpha}{\zeta}$ \\
\hline $\begin{array}{l}\text { Projectives } \\
\text { for } E\end{array}$ & $\begin{array}{c}\Delta(\alpha) \\
\Delta(\zeta) \Delta(\delta) \\
\Delta(\zeta)\end{array}$ & $\begin{array}{l}\Delta(\beta) \\
\Delta(\epsilon)\end{array}$ & $\Delta(\gamma)$ & $\begin{array}{l}\Delta(\delta) \\
\Delta(\zeta)\end{array}$ & $\Delta(\epsilon)$ & $\Delta(\zeta)$ \\
\hline
\end{tabular}

Table of Mackey functors for $C_{4}$ and modules for the stratified algebra $E$ 
We next present the relative Auslander-Reiten quivers for the categories $\mathcal{D}$. In the quiver for $C_{4}$ the Mackey functors ${ }_{4}^{4}$ at the corners should be identified with the third copy of this Mackey functor in the middle of the opposite side, and similarly with the copies of the Mackey functor ${ }_{4}^{2}$. There are also some further identifications which should be made in cases when a single functor appears more than once in the diagram. We show only the sink and source maps which form part of relative almost split sequences, not the other sink and source maps involving Ext-projectives and Ext-injectives. Where there is no almost split sequence at a particular mesh in the diagram this is indicated with the symbol $\diamond$. This happens because the Mackey functor on the left is Ext-injective and the one on the right is Ext-projective. In the case of the Mackey functors which are the projective covers of 1 and 2 we have omitted $\diamond$. These functors are both projective and injective.

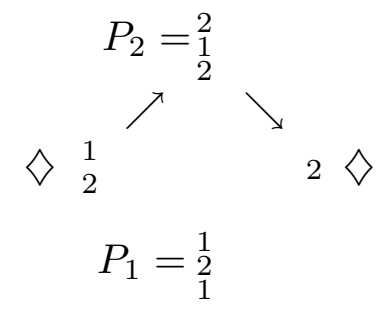

The relative Auslander-Reiten quiver of functors with $\Delta$-filtration for $C_{2}$ at $p=2$. 


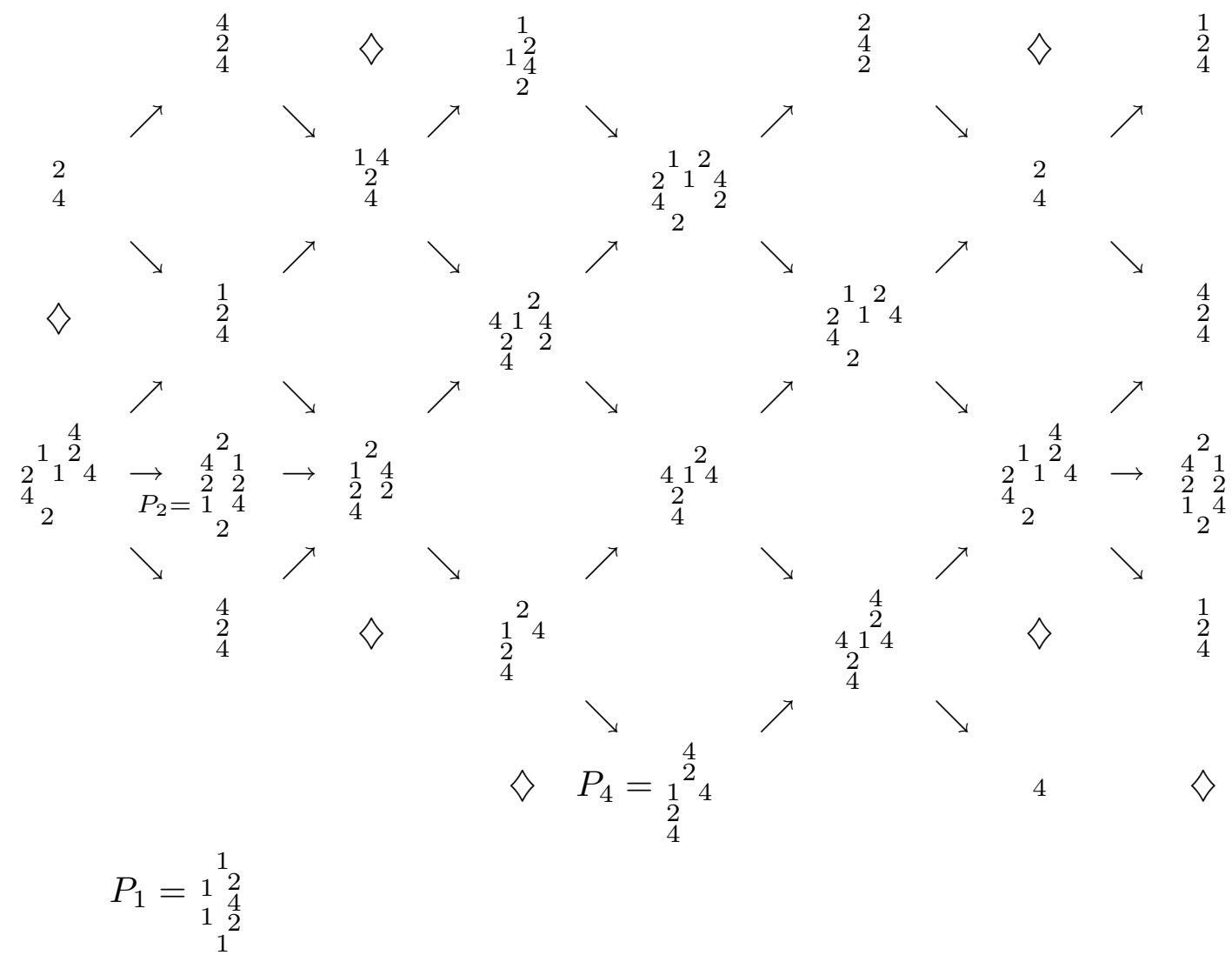

The relative Auslander-Reiten quiver of functors with $\Delta$-filtration for $C_{4}$ at $p=2$.

To explain the calculations which produce these diagrams we will concentrate on Mackey functors for the group $C_{4}$. The case of $C_{2}$ is much easier, and we leave it to the reader. We start with the functors $\Delta_{H, U}$ where $U$ is a $p$-permutation module. The possibilities for $H, U$ are listed at the top of the first table. The structures of $\Delta_{1, R}=F Q_{R}$, $\Delta_{C_{2}, R}=\operatorname{Inf}_{C_{4} / C_{2}}^{C_{4}} F Q_{R}$ and $\Delta_{C_{4}, R}=\operatorname{Inf}_{C_{4} / C_{4}}^{C_{4}} F Q_{R}$ may be deduced by inflating the result of $[14,15.3]$. Next $\Delta_{C_{2}, R}=\operatorname{Inf}_{C_{4} / C_{2}}^{C_{4}} F Q_{R}$ is the inflation of a projective functor for $C_{4} / C_{2}$ by $[14,13.3]$, and we know its structure from the tables at the end of [14]. The most difficult functors $\Delta_{H, U}$ to determine are $\Delta_{1, R}$ and $\Delta_{1, R C_{4}}$. By $[14,13.3]$ we know that $\Delta_{1, R C_{4}}=P_{1, R}$ is the projective cover of 1 . Since $U \rightarrow F Q_{U}$ is a right exact functor, the module epimorphism $R C_{4} \rightarrow{ }_{R}^{R}$ gives an epimorphism $\Delta_{1, R C_{4}} \rightarrow \Delta_{1, R}$. We deduce that $\Delta_{1, R}$ has 1 as its unique simple quotient. By computing dimensions of fixed quotients we see that the composition factors of $\Delta_{1, R}$ are $1,1,2,2$ and 4 . The exact sequence of modules $0 \rightarrow R \rightarrow{ }_{R}^{R} \rightarrow R \rightarrow 0$ gives an exact sequence $\Delta_{1, R} \rightarrow \Delta_{1,{ }_{R}} \rightarrow \Delta_{1, R} \rightarrow 0$ and since we know the composition factors of each term we see that there is a short exact sequence of functors $0 \rightarrow{ }_{2}^{1} \rightarrow \Delta_{1, R} \rightarrow{ }_{4}^{1} \rightarrow 0$. By [14, 12.4], $\Delta_{1, R}^{*} \cong F P_{R}$ is an image 
of the projective $P_{C_{2}, R}$ so has 2 as its unique simple quotient. Hence the socle of $\Delta_{1, R}$ is 2. The subfunctor of $\Delta_{1, R}$ generated by its value at 2 is zero on the identity group since the operation $R_{1}^{C_{2}}$ is zero (because $C_{2}$ acts trivially on ${ }_{R}^{R}$ ). Hence there is a short exact sequence $0 \rightarrow{ }_{2}^{2} \rightarrow \Delta_{1, R} \rightarrow{ }_{1}^{1} \rightarrow 0$. From all this we deduce that $\Delta_{1, R}$ has the stated form.

After this, the structure of $P_{1, R}=\Delta_{1, R C_{4}}$ (also denoted $P_{1}$ in the tables) is relatively easy to determine. The dimensions of this functor at the various subgroups give the composition factor multiplicities, and they are as presented. The functor is self-dual, with top and socle isomorphic to $1[14,13.3]$. Its value at the identity group is $R C_{4}$, which is uniserial with four 1 factors. Thus no sections of the form $1 \oplus 1$ appear. There is an epimorphism $\Delta_{1, R C_{4}} \rightarrow \Delta_{1, R}$ whose codomain we have already determined. By looking at the composition factors we have a short exact sequence $0 \rightarrow{ }_{1}^{1} \rightarrow \Delta_{1, R C_{4}} \rightarrow \Delta_{1,{ }_{R}^{R}} \rightarrow 0$. Combining these ingredients gives the form of the functor.

As a next step we compute the structure of the indecomposable projective Mackey functors. We have just done $P_{1, R}$. We know the Cartan invariants of these functors by the method of [14], and also by $[14,19.1]$ we know that $P_{C_{2}, R}$ is self-dual with socle 2 . Its value at the identity group is $P_{C_{2}, R}(1)={ }_{R}^{R}$ by $[14,12.7]$ and so $F Q_{R}=\Delta_{1, R}$ is a subfunctor by $[14,12.4]$. In fact this is the subfunctor generated by the value at the identity group, so it is the start of a $\Delta$-filtration by 6.1 . Thus the quotient by this subfunctor has a filtration by $\Delta$ 's (whose general form we have already determined) and has composition factors 2 , 2 , and 4 . It follows that we have a short exact sequence $0 \rightarrow \Delta_{1, R} \rightarrow P_{C_{2}, R} \rightarrow \Delta_{C_{2}, R}{ }_{R} \rightarrow 0$ since this is the only possibility with these composition factors. Putting this together gives the structure presented for $P_{C_{2}, R}$.

We may follow a similar line of argument for $P_{C_{4}, R}$. We know its composition factors, that $P_{C_{4}, R}(1)=R$ and so $\Delta_{1, R}$ is the start of a $\Delta$-filtration of $P_{C_{4}, R}$. The quotient has composition factors 2,4 and 4 so the $\Delta$-filtration must have factors $\Delta_{C_{2}, R}={ }_{4}^{2}$ and $\Delta_{C_{4}, R}=4$. Since $P_{C_{4}, R}$ has 4 as its unique simple quotient we deduce that there is a short exact sequence

$$
0 \rightarrow \Delta_{1, R} \rightarrow P_{C_{4}, R} \rightarrow \stackrel{4}{2} \underset{4}{2} \rightarrow 0
$$

where the right-hand functor is uniserial. Also by [14, 12.4], $F P_{R}={ }_{1}^{4}$ is an image of $P_{C_{4}, R}$. From all this the structure follows.

The next step is to compute the groups $\operatorname{Ext}\left(\Delta_{H, U}, \Delta_{K, W}\right)$ where $U$ and $W$ are $p$ permutation modules. It speeds up the work to use Theorem 5.1, which implies that we can only get a non-zero Ext group when $(K, W)=(1, R),\left(1,{ }_{R}^{R}\right)$ or $\left(C_{2}, R\right)$. By direct calculation with projective presentations we find that $\operatorname{Ext}\left(\Delta_{H, U}, \Delta_{1, R}\right)=R$ when $(H, U)=\left(C_{2}, R\right),\left(C_{2},{ }_{R}^{R}\right)$ or $\left(C_{4}, R\right), \operatorname{Ext}\left(\Delta_{C_{2}, R}, \Delta_{1, R}\right)=R$ and $\operatorname{Ext}\left(\Delta_{C_{4}, R}, \Delta_{C_{2}, R}\right)=R$. All other Ext groups between $\Delta$ 's are zero.

At this point we may directly construct the Ext-injective hull $T_{H, U}$ of each $\Delta_{H, U}$ by the method of [10, Lemma 4]. The method is to start with a Mackey functor $M=M_{0}$ 
(we do not mean an ascending filtration here) and work along the list of $\Delta_{H, U}$ in such a way that all $\Delta_{K, W}$ with $K$ conjugate to a subgroup of $H$ are considered prior to $\Delta_{H, U}$. At each stage we have a functor $M_{r}$ and we form a maximal extension

$$
0 \rightarrow M_{r} \rightarrow M_{r+1} \rightarrow \Delta_{H, U}^{n} \rightarrow 0
$$

where $n=\operatorname{dim} \operatorname{Ext}\left(\Delta_{H, U}, M_{r}\right)$ and the extension corresponds to a basis for this Ext group. We now repeat the process with $M_{r+1}$ instead of $M_{r}$, using the next $\Delta_{H^{\prime}, U^{\prime}}$ in the list. We finish this process with a monomorphism from the original functor $M$ to an Ext-injective functor. Selecting a minimal direct summand of this in which $M$ embeds gives the Extinjective hull of $M$.

For example, if we wish to find the Ext-injective hull of $\Delta_{1, R}={ }_{2}^{1}$ the first non-zero $\operatorname{group} \operatorname{Ext}\left(\Delta_{H, U}, \begin{array}{l}1 \\ 4\end{array}\right)$ occurs with $(H, U)=\left(C_{2}, R\right)$, in which case $\Delta_{C_{2}, R}={ }_{4}^{2}$. We compute $\operatorname{Ext}\left(\begin{array}{c}2 \\ 4\end{array}, \begin{array}{l}1 \\ 4\end{array}\right)=R$ with non-split extension $M_{1}=\frac{1}{2}_{4}^{2}{ }_{4}^{4}$. Now $\operatorname{Ext}\left(\Delta_{C_{2},{ }_{R}}, M_{1}\right)=0$, and we move on to $\operatorname{Ext}\left(\Delta_{C_{4}, R}, M_{1}\right)=R^{2}$. The maximal extension here is

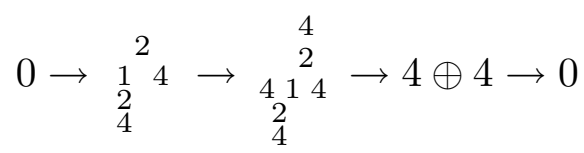

and the middle term is the Ext-injective hull of $\Delta_{1, R}$. By means of the dual construction we also determine the Ext-projective covers of the $\Delta_{H, U}$.

We now construct all the remaining objects in $\mathcal{D}$. In the first instance this was done by trial and error, knowing that the ascending filtration of each object refines to a $\Delta$ filtration so that arbitrary objects may be constructed by successively extending by direct sums $\Delta_{H, U}^{n}$. Given the above display of objects in $\mathcal{D}$ by means of the relative AuslanderReiten quiver, we see immediately that these objects are indeed Mackey functors with a $\Delta$-filtration. By computing all possible dimensions of homomorphisms between the Mackey functors listed (an $18 \times 18$ matrix in the case of $C_{4}$ ) we verify that all of the short exact sequences presented do satisfy the lifting property of an almost split sequence with respect to homomorphisms between the Mackey functors that we have found. A standard argument now shows that we have constructed a union of connected components of the quiver. To see that there are no more objects in $\mathcal{D}$ it remains to show that there are no more connected components of the quiver. We have failed to find an argument which justifies this final step, except to say by inspection that there are no more objects in $\mathcal{D}$.

As regards our description of the standard modules and indecomposable projectives for $E$, we have first labeled the simple $E$-modules $\alpha, \beta, \gamma, \delta, \epsilon, \zeta$ arbitrarily. These modules biject with the indecomposable summands $T_{H, U}$ of $T$. The standard modules have the form $\operatorname{Hom}_{\mu_{R}(G)}\left(\Delta_{H, U}, T\right)$ and their structure is determined by explicit calculation of these homomorphisms, and an examination of how they factor through homomorphisms between the summands of $T$. Finally, the filtrations of the projective $E$-modules are obtained from the construction of these filtrations at the end of the proof of Theorem 9.1 


\section{Characterizations of objects in $\mathcal{D}$ and structure of the Auslander-Reiten quiver}

Part of our motivation for this study has been the tantalizing possibility that the framework we have constructed may shed light on Alperin's weight conjecture [2]. We recall that when $R$ is a field of positive characteristic $p$. Alperin defines a weight to be a pair $(H, U)$ where $H$ is a $p$-subgroup of $G$ and $U$ is a block of defect zero for $N_{G}(H) / H$, that is, a simple projective $R\left[N_{G}(H) / H\right]$-module. Such a module is in particular a $p$ permutation module, and so the weights form a subset of the indexing set $\Lambda$ which we have been considering.

There is a categorical property which distinguishes within $\mathcal{D}$ the functors $\Delta_{H, U}$ where $(H, U)$ is a weight, namely, they are the objects of $\mathcal{D}$ which are simple as objects of the larger category $\operatorname{Mack}_{R}(G)$. Furthermore they are Ext-injective in $\mathcal{D}$, so that they occur on the right-hand edge of the relative Auslander-Reiten quiver of $\mathcal{D}$. We obtain also in this section a characterization of all the functors $\Delta_{H, U}$ which are Ext-injective as the ones for which $U$ is a projective $R\left[N_{G}(H) / H\right]$-module. These also coincide with the functors satisfying $\Delta_{H, U} \cong \nabla_{H, U}$. We show finally in this section that the relative Auslander-Reiten quiver of $\mathcal{D}$ is compatible with ascending filtrations, in a sense described in Proposition 11.3. We assume throughout this section that $R$ is a field of characteristic $p$.

(11.1) PROPOSITION. Suppose that $R$ is a field of characteristic $p$ and let $U$ be an $R\left[N_{G}(H) / H\right]$-module. Then

(a) $U$ is a projective $R\left[N_{G}(H) / H\right]$-module if and only if $\Delta_{H, U} \cong \nabla_{H, U}$, and

(b) $(H, U)$ is a weight if and only if $H$ is a p-group and $\Delta_{H, U}$ is a simple Mackey functor.

Proof. (a) By $[14,13.1]$ we have that $U$ is projective if and only if $F P_{U} \cong F Q_{U}$, which immediately implies $\Delta_{H, U} \cong \nabla_{H, U}$. On the other hand, if $\Delta_{H, U}^{G} \cong \nabla_{H, U}^{G}$ then $\Delta_{H, U}^{G} \downarrow_{N_{G}(H)} \cong \nabla_{H, U}^{G} \downarrow_{N_{G}(H)}$ and so the subfunctors of these two functors generated by their values at $H$ are isomorphic. By Corollary 4.4 the subfunctor on the left is isomorphic to $\Delta_{H, U}^{N_{G}(H)}$. On the right by a similar argument to the proof of 4.4 we have

$$
\begin{aligned}
\nabla_{H, U}^{G} \downarrow_{N_{G}(H)}^{G} & =\nabla_{H, U}^{N_{G}(H)} \uparrow_{N_{G}(H)}^{G} \downarrow_{N_{G}(H)}^{G} \\
& \cong \bigoplus_{x \in\left[N_{G}(H) \backslash G / N_{G}(H)\right]}{ }^{x}\left(\nabla_{H, U}^{N_{G}(H)} \downarrow_{N_{G}(H)^{x} \cap N_{G}(H)}\right) \uparrow_{N_{G}(H) \cap{ }^{x} N_{G}(H)}^{N_{G}(H)}
\end{aligned}
$$

and the summand corresponding to $x=1$ is isomorphic to $\nabla_{H, U}^{N_{G}(H)}$. Since $\nabla_{H, U}^{N_{G}(H)}(H) \cong$ $\nabla_{H, U}^{G}(H) \cong U$ it follows that all the other summands in the direct sum are zero at $H$. Hence the subfunctor of $\nabla_{H, U}^{G} \downarrow_{N_{G}(H)}^{G}$ generated by the value at $H$ is a subfunctor of the summand $\nabla_{H, U}^{N_{G}(H)}$. This shows that $\Delta_{H, U}^{N_{G}(H)}$ is isomorphic to a subfunctor of $\nabla_{H, U}^{N_{G}(H)}$.

From this it follows that $F Q_{U}$ is isomorphic to a subfunctor of $F P_{U}$, since the previous

two functors are inflated from these. We deduce that all the restriction maps $R_{J}^{L}$ are 
injective for $F Q_{U}$, since the same is true for $F P_{U}$. It is well known that this condition is equivalent to the projectivity of $U$.

(b) If $(H, U)$ is a weight then $H$ is a $p$-group and by $[14,13.1$ (vii)] and $[13,8.1$ (ii)] we have that $\Delta_{H, U}=S_{H, U}$ is simple. On the other hand if $\Delta_{H, U}$ is simple then its value $U$ at its minimum subgroup $H$ must be a simple module by the parametrization of simple functors in [13], and by [13, 8.1(ii)] and exactness of induction and inflation we have that $F Q_{U}$ must be a simple Mackey functor for $N_{G}(H) / H$. It follows from [14, 13.1] (using the condition dual to (vii) of that proposition) that $U$ must be a projective $R\left[N_{G}(H) / H\right]$-module.

We now characterize those $\Delta_{H, U}$ which are Ext-injective, and those which are Extprojective.

(11.2) PROPOSITION. Suppose that $R$ is a field of characteristic $p$.

(a) Let $U$ be a projective $R\left[N_{G}(H) / H\right]$-module. Then

$$
\operatorname{Ext}\left(\Delta_{K, W}, \Delta_{H, U}\right)=0 \quad \text { and } \quad \operatorname{Ext}\left(\Delta_{H, U}, \nabla_{K, W}\right)=0
$$

for all pairs $(K, W)$ where $W$ is a $p$-permutation $R\left[N_{G}(K) / K\right]$-module. In particular, $\Delta_{H, U}$ is Ext-injective in $\mathcal{D}$.

(b) Conversely, if $U$ is a p-permutation $R\left[N_{G}(H) / H\right]$-module and $\operatorname{Ext}\left(\Delta_{K, W}, \Delta_{H, U}\right)=0$ for all pairs $(K, W)$ where $W$ is a p-permutation $R\left[N_{G}(K) / K\right]$-module then $U$ is projective.

Proof. (a) We know from Theorem 5.1 that $\operatorname{Ext}\left(\Delta_{K, W}, \Delta_{H, U}\right)$ can only be non-zero when $K>_{G} H$. On the other hand if $U$ is a projective module then $\Delta_{H, U} \cong \nabla_{H, U}$ by 11.1 and $\operatorname{Ext}\left(\Delta_{K, W}, \nabla_{H, U}\right)$ can only be non-zero when $K=_{G} H$. We deduce that the Ext group is always zero.

Similarly, $\operatorname{Ext}\left(\Delta_{H, U}, \nabla_{K, W}\right)$ is only non-zero when $H={ }_{G} K$. But again $\Delta_{H, U} \cong \nabla_{H, U}$ and $\operatorname{Ext}\left(\nabla_{H, U}, \nabla_{K, W}\right)$ is only non-zero when $H<_{G} K$, so the Ext group is always zero.

(b) We suppose without loss of generality that $U$ is indecomposable. Let us write $N=N_{G}(H)$ and suppose that $K$ is a subgroup of $G$ with $H \leq K \leq N$. Let $V$ be a $p$ permutation $N_{N}(K) / K$-module. Then by $4.1 \Delta_{K, V}^{N} \uparrow_{N}^{G}$ is a direct sum of functors $\Delta_{K, W}$ with $W$ a $p$-permutation $R\left[N_{G}(K) / K\right]$-module and so

$$
\operatorname{Ext}\left(\Delta_{K, V}^{N} \uparrow_{N}^{G}, \Delta_{H, U}^{G}\right) \cong \operatorname{Ext}\left(\Delta_{K, V}^{N}, \Delta_{H, U}^{G} \downarrow_{N}^{G}\right)=0
$$

Since $\Delta_{H, U}^{N}$ is a summand of $\Delta_{H, U}^{G} \downarrow_{N}^{G}$ by 4.4 we deduce that $\operatorname{Ext}\left(\Delta_{K, V}^{N}, \Delta_{H, U}^{N}\right)=0$ for all p-permutation $N_{N}(K) / K$-modules $V$.

Now consider the short exact sequence of Mackey functors for $N_{G}(H) / H$

$$
0 \rightarrow F Q_{U} \rightarrow P \rightarrow X \rightarrow 0
$$


where $P$ is the indecomposable projective Mackey functor on $N_{G}(H) / H$ with $P(H / H)=U$ and the morphism from $F Q_{U}$ to $P$ extends the identity map on the evaluation at $H / H$. This morphism is considered in $[14,12.4]$, where it is shown that it is a monomorphism. We define $X$ to be the cokernel of this morphism. In fact $F Q_{U}=\Delta_{H / H, U}^{N / H}$ and the image of $F Q_{U}$ in $P$ is $I_{\{H / H\}} P$ since it is generated by the value of $P$ at the identity subgroup $H / H$. It follows from 7.1 and 6.1 that this is the start of a filtration of $P$ by $\Delta$ functors, which will have the form $\Delta_{K / H, W}^{N / H}$ with $H<K \leq N$. Inflating this sequence to $N$ gives an extension $0 \rightarrow \Delta_{H, U}^{N} \rightarrow \operatorname{Inf}_{N / H}^{N} P \rightarrow \operatorname{Inf}_{N / H}^{N} X \rightarrow 0$ where now by $4.7 \operatorname{Inf}_{N / H}^{N} X$ has a filtration by functors $\Delta_{K, W}^{N}$ with $H<K \leq N$ and it follows that $\operatorname{Ext}\left(\operatorname{Inf}_{N / H}^{N} X, \Delta_{H, U}^{N}\right)=0$. Thus the sequence splits. Since $P$ and $\operatorname{Inf}_{N / H}^{N} P$ are indecomposable it follows that $F Q_{U} \cong P$. Now by $[14,13.1]$ it follows that $U$ is projective.

(11.3) PROPOSITION. Suppose that $R$ is a field of characteristic $p$. Then $\Delta_{H, U}$ is Ext-projective in $\mathcal{D}$ if and only if $H=O^{p}(H)$.

Proof. By the equivalence of categories in $[14,10.1]$ and the reduction process indicated at the start of Theorems 5.1 and 7.1 it suffices to assume $H$ is a $p$-group, and we will now do this.

Suppose that $\Delta_{H, U}$ is Ext-projective with $1 \neq H$ a $p$-subgroup of $G$, and let $K \subset H$ be a subgroup of index $p$ in $H$. Then using Lemmas 4.1 and 4.3 we have

$$
\begin{aligned}
0 & =\operatorname{Ext}\left(\Delta_{H, U}^{G}, \Delta_{K, R}^{H} \uparrow_{H}^{G}\right) \\
\cong & \operatorname{Ext}\left(\Delta_{H, U}^{G} \downarrow_{H}^{G}, \Delta_{K, R}^{H}\right) \\
& =\operatorname{Ext}\left(\Delta_{H, U}^{H}, \Delta_{K, R}^{H}\right) .
\end{aligned}
$$

It follows that $\operatorname{Ext}\left(\Delta_{H, R}^{H}, \Delta_{K, R}^{H}\right)=0$ since $U$ is now considered as a module for the trivial group $H / H$ and so is a direct sum of copies of $R$. However, $\Delta_{H, R}^{H}=\operatorname{Inf}_{H / K}^{H} S_{H / K, R}^{H / K}$ where $S_{H / K, R}^{H / K}$ is a simple functor, and $\Delta_{K, R}^{H}=\operatorname{Inf}_{H / K}^{H} F Q_{R}$. From the methods of [14] applied to the cyclic group $H / K$ of order $p$ we see that there is a short exact sequence

$$
0 \rightarrow F Q_{R} \rightarrow P_{H / K, R} \rightarrow S_{H / K, R} \rightarrow 0
$$

where $P_{H / K, R}$ is the projective cover of $S_{H / K, R}$, and this sequence is not split. Inflating it to $H$ gives a non-split extension which contradicts our previous calculation that the Ext group was 0 . Hence if $\Delta_{H, U}$ is Ext-projective it cannot happen that $H \neq 1$.

Conversely if $H=1$ then by 5.1 we know $\operatorname{Ext}\left(\Delta_{H, U}, \Delta_{K, W}\right)=0$ for all $(K, W)$, and so $\Delta_{H, U}$ is Ext-projective. 
We show now that the relative almost split sequences in $\mathcal{D}$ are compatible with ascending filtrations in that the terms of an ascending filtration of the Mackey functors in an almost split sequence also form an exact sequence, which is split most of the time.

(11.4) PROPOSITION. Suppose that $R$ is a field of characteristic $p$. Let $1=$ $H_{1}, H_{2}, \ldots, H_{n}=G$ be a list of representatives of the conjugacy classes of subgroups of $G$ with the property that whenever $H_{i}$ is conjugate to a subgroup of $H_{j}$ then $i \leq j$. For each Mackey functor $M$ consider the terms $M_{i}=I_{\left\{H_{1}, \ldots, H_{i}\right\}} M$ of the corresponding ascending filtration. Let $0 \rightarrow A \rightarrow B \rightarrow C \rightarrow 0$ be a relative almost split sequence of Mackey functors in $\mathcal{D}$.

(a) For every $i$ the sequence $0 \rightarrow A_{i} / A_{i-1} \rightarrow B_{i} / B_{i-1} \rightarrow C_{i} / C_{i-1} \rightarrow 0$ is split exact.

(b) For every $i$ the sequence $0 \rightarrow A_{i} \rightarrow B_{i} \rightarrow C_{i} \rightarrow 0$ is exact, and is split if $C_{i} \neq C$.

(c) For every $i$ the sequence $0 \rightarrow A / A_{i} \rightarrow B / B_{i} \rightarrow C / C_{i} \rightarrow 0$ is exact, and is split if $A_{i} \neq 0$.

Proof. We first note that by 6.1 (a) if $M$ is in $\mathcal{D}$ then so is every $M_{i}$ and $M / M_{i}$. The exactness of the sequences in (b) is evidently equivalent to the exactness of the sequences in (c). From the definitions we see that whenever $\eta: M \rightarrow N$ is a morphism of Mackey functors then for every $i$ the restriction $\eta_{i}: M_{i} \rightarrow N_{i}$ is a morphism. If $\eta$ is a monomorphism then so is $\eta_{i}$ and if $\eta$ is an epimorphism then so is $\eta_{i}$.

Consider now the almost split sequence $0 \rightarrow A \rightarrow B \rightarrow C \rightarrow 0$. Suppose for some $i$ that $C_{i} \neq C$. Then the inclusion $C_{i} \rightarrow C$ is not split epi, it factors $C_{i} \rightarrow B \rightarrow C$, and hence we obtain a factorization of the identity $C_{i} \rightarrow B_{i} \rightarrow C_{i}$. Thus $B_{i}=C_{i} \oplus K$ where $K=\operatorname{Ker}\left(B_{i} \rightarrow C_{i}\right)$. We have $A_{i} \subseteq K$ and from the last direct sum we deduce that $K=K_{i}$. Hence $A_{i}=K$, and $B_{i}=C_{i} \oplus A_{i}$. We deduce simultaneously that $0 \rightarrow A_{i} \rightarrow B_{i} \rightarrow C_{i} \rightarrow 0$ is exact and split.

By the dual argument if $A_{i} \neq 0$ then the morphism $A \rightarrow A / A_{i}$ is not split mono and factors as $A \rightarrow B \rightarrow A / A_{i}$, from which we obtain a factorization of the identity $A / A_{i} \rightarrow B / B_{i} \rightarrow A / A_{i}$. Thus $B / B_{i}=A / A_{i} \oplus B / L$ where $B / L=\operatorname{Coker}\left(A / A_{i} \rightarrow B / B_{i}\right)$, i.e. $L=A+B_{i}$. Now $B / L$ has $C / C_{i}$ as an image and is an image of $C$. We deduce from the direct sum that $(B / L)_{i}=0$. Since $C / C_{i}$ is the largest quotient of $C$ with $\left(C / C_{i}\right)_{i}=0$ we deduce that $B / L \cong C / C_{i}$, and hence $B / B_{i}=A / A_{i} \oplus C / C_{i}$. Again we deduce that $0 \rightarrow A / A_{i} \rightarrow B / B_{i} \rightarrow C / C_{i} \rightarrow 0$ is exact and split.

We now claim that the conditions $A_{i}=0$ and $C_{i}=C$ cannot simultaneously happen, from which we will deduce that $0 \rightarrow A_{i} \rightarrow B_{i} \rightarrow C_{i} \rightarrow 0$ is always exact. For if $A_{i}=0$ then by 6.1 (a) every $\Delta$-factor of $A$ has the form $\Delta_{H_{j}, U_{j, l}}$ with $j \geq i$, and if $C_{i}=C$ then every $\Delta$-factor of $C$ has the form $\Delta_{H_{k}, U_{k, l}}$ with $k \leq i$. Since $\operatorname{Ext}\left(\Delta_{H_{k}, U_{k, l}}, \Delta_{H_{j}, U_{j, l}}\right)=0$ if $j \geq k$ we would deduce that $0 \rightarrow A \rightarrow B \rightarrow C \rightarrow 0$ is split. As this is not the case we have established the claim.

All of the sequences $0 \rightarrow A_{i} \rightarrow B_{i} \rightarrow C_{i} \rightarrow 0$ are now known to be exact, and it follows that the sequences $0 \rightarrow A_{i} / A_{i-1} \rightarrow B_{i} / B_{i-1} \rightarrow C_{i} / C_{i-1} \rightarrow 0$ are all exact. Since all terms are direct sums of functors $\Delta_{H_{i}, U_{i, l}}$, these latter sequences split by 5.1 . 


\section{REFERENCES}

[1] A. Adem and D. Karagueuzian, Essential cohomology of finite groups, Commentarii Mathematici Helvetici 72 (1997), 101-109.

[2] J.L. Alperin, Weights for finite groups, Proc. Symp. Pure Math. 47 (1987), 369-379.

[3] S. Bouc, Résolutions de foncteurs de Mackey, Proc. Symp. Pure Math. 63 (1998), 31-84.

[4] E. Cline, B. Parshall and L. Scott, Stratifying endomorphism algebras, Mem. Amer. Math. Soc. 124 (1996), no. 591.

[5] C.W. Curtis and I. Reiner, Methods of representation theory II, Wiley 1987.

[6] A.W.M. Dress, Contributions to the theory of induced representations, in ed H. Bass, Algebraic K-Theory II, Lecture Notes in Math. 342 (183-240), Springer-Verlag (1973).

[7] P.J. Hilton and U. Stammbach, A course in homological algebra, Springer-Verlag 1971.

[8] L.G. Lewis, Jr., The theory of Green functors, unpulished notes, 1980.

[9] F. Luca, Mackey functors with chain conditions, J. Algebra 205 (1998), 661-698.

[10] C.M. Ringel, The category of modules with good filtrations over a quasi-hereditary algebra has almost split sequences, Math. Zeit. 208 (1991), 209-225.

[11] J. Thévenaz, Some remarks on $G$-functors and the Brauer morphism, J. reine angew. Math. 384 (1988), 24-56.

[12] J. Thévenaz, G-Algebras and modular representation theory, Oxford University Press 1995.

[13] J. Thévenaz and P.J. Webb, Simple Mackey functors, in: Proc. of 2nd international group theory conference, Bressanone (1989), Supplemento ai Rendiconti del Circolo Matematico di Palermo 23 (1990), 299-319.

[14] J. Thévenaz and P.J. Webb, The structure of Mackey functors, Trans. Amer. Math. Soc. 347 (1995), 1865-1961.

[15] P.J. Webb, A split exact sequence of Mackey functors, Commentarii Math. Helvetici 66 (1991), 34-69. 